\title{
Transform Domain Processing Based Cooperative Spectrum Sharing Protocol with Adaptive Relaying
}

\author{
Wu Xuanli, Member, IEEE, Han Xingling, Deng Xiao, Fabrice Labeau, Senior Member, IEEE, and Han \\ Shuai, Member, IEEE
}

\begin{abstract}
In this paper, we propose a two-time slot cooperative spectrum sharing protocol employing transform domain processing technology, in which primary users and secondary users adopt sinusoidal signals and chirp signals, respectively. Moreover, an adaptive relaying mechanism is used in our protocol to improve the flexibility of cooperation. Then, a Newton iteration method based sub-optimal power allocation algorithm is presented to reduce the complexity of exhaustive method while guaranteeing the outage probability constraint of the primary system. After that, a secondary user selection algorithm which focuses on the statistical channel conditions is proposed to further improve the performance of spectrum sharing. Simulation results show that compared with the existing protocol, outage probability, achievable data rate and average energy efficiency are significantly improved for the secondary system in our proposed protocol. Moreover, lower outage probability, higher achievable data rate and average energy efficiency of the secondary system can be realized with an increase in the number of secondary users.
\end{abstract}

Index Terms - cognitive radio, cooperative spectrum sharing, Fractional Fourier Transform (FrFT), outage probability

\section{INTRODUCTION}

$\mathrm{W}$ ITH the rapid development of wireless communications, wireless spectrum has become an essential resource in the society and many literatures have focused on the efficient use of spectrum resources [1-2]. In order to utilize spectrum resources more efficiently, Cognitive Radio (CR) technology is proposed to solve the unbalanced spectrum resource utilization problem [3]. As a key technology of cognitive radio system, spectrum sharing, which aims to realize efficient and reliable communications of secondary users through sharing spectrum

Manuscript received March 14th, 2017.

Wu Xuanli, Han Xingling, Deng Xiao and Han Shuai are with School of Electronic and Information Engineering, Harbin Institute of Technology, Harbin, China (email: xlwu2002@hit.edu.cn, hanxingling1993@126.com, 1120510402@hit.edu.cn, hanshuai@hit.edu.cn,); Fabrice Labeau is with Department of Electrical and Computer Engineering, McGill University, Quebec, Canada (email: fabrice.labeau@mcgill.ca).

This work has been partially supported by the National Basic Research Program of China (973 Program), under grant No. 2013CB329003, National Nature Science Foundation of China under grant No. 61301100 and Hydro-Quebec, the Natural Sciences and Engineering Research Council of Canada, and McGill University in the framework of the NSERC/Hydro-Quebec Industrial Research Chair in Interactive Information Infrastructure for the Power Grid (IRCPJ406021-14) between primary users and secondary users, on the premise that communication requirements of primary users are satisfied, has very broad application prospects in the future wireless communication networks. Moreover, as a combination of cooperative communications and spectrum sharing technology, cooperative spectrum sharing, which can fully achieve diversity gain through the cooperation between primary users and secondary users to improve spectrum efficiency of cognitive radio system and satisfy the requirement of spectrum sharing, has attracted more attention [4-5].

In cooperative spectrum sharing, secondary user can perform as a cooperative relay to play an active role in the communications of primary user. As a reward, the secondary user can obtain the opportunity to access the system spectrum while guaranteeing the communication quality of primary user. References [6-7] propose different two-time slot spectrum sharing protocols, which employ Amplify and Forward (AF) and Decode and Forward (DF) mechanism, respectively. The secondary user who acts as the relay will allocate its power to forward the signals of primary user to primary receivers and then in the second time slot, the signals of secondary user will be transmitted to secondary receiver. However, in reality, the channel condition varies quickly because of channel fading. Thus, choosing a fixed mechanism, i.e., DF or AF, is not suitable for the fast fading channels. To overcome this obstacle, the adaptive relaying protocols which adopt different mechanism, i.e., $\mathrm{AF}$ and $\mathrm{DF}$ mechanism, in different channel conditions are proposed [8-9]. Furthermore, ref. [8] also proposes a power allocation algorithm to improve system performance in terms of lower outage probability of the secondary system. However, they do not consider the scenario that multiple alternative relays exist. Ref. [10] selects the best relay according to the channel conditions between the secondary transmitter and the primary receiver. Ref. [11] proposes a relay selection strategy under the circumstances that only outdated CSIs is available and both outdated CSIs and statistical channel information are available, respectively. However, they do not consider the channel status of different transmission links so that the performances of secondary user cannot be guaranteed.

To our best knowledge, in cooperative spectrum sharing scenario, one essential problem is how to distinguish the 
primary signal and the secondary signal occupying the same bandwidth and time slot at the receiver without bringing serious interference between each other. There exist several methods to alleviate such kind of interference. The first one is to divide transmission time slot. Specifically, during the second time slot, we can further divide this time slot into two sub-time slot parts. Thus, there are totally three different length time slots in one whole transmission period. In the first sub-time slot, the relay could assist the primary user while in the second sub-time slot; they could share the same bandwidth with primary user to transmit their own signals [12-13]. The second way is to divide licensed bandwidth, which means that the relay will use different bandwidth to transmit its own signals and the primary signals respectively during the second transmission time slot [14-15]. However, the spectrum efficiency will be reduced for the abovementioned references. There indeed exist other methods to reduce the interference without destroying the structure of transmission time slot and bandwidth. For example, in ref. [16], STBC is used at the relay and in ref. [17], NOMA and SIC are used in the relay and the receiver. However, in the STBC method will decrease the data rate of secondary users, and the realization complexity is extremely high for SIC algorithm when the number of primary users waiting for decoding their transmission information is large. In order to reduce the interference to primary users, spread spectrum based spectrum sharing strategies are also proposed in [18] and [19], and the signals of secondary user are spread over the whole transmission frequency bandwidth with power control. Although the interference to primary users can be reduced, the transmission data rate of secondary users will be reduced and the interference from primary users to secondary users may be very huge in cooperation mode. Hence, we need to find a suitable method to reduce such interference and achieve higher spectrum efficiency with moderate realization complexity.

Several applications like Synthetic Aperture Radar (SAR) and chirp spread spectrum (CSS) technique uses chirp signals. Owing to the recent advance of Surface Wave Acoustic (SAW) technology, generating wide-band chirp pulses become easier and make the use of chirp signals on abovementioned techniques more efficient. As chirp signals are widely used in radar, sonar and signal processing fields, estimation of their parameters such as amplitude, chirp rate, initial frequency and initial phase is an important problem [20].

FrFT is a useful time-frequency tool which is very suitable for signal processing, especially for detection and parameter estimation of chirp signal. Ref. [21] utilizes FrFT to measure parameters in ultrasonic range system while [21] proposes an FrFT based algorithm to estimate the parameters of the moving target. In the Fractional Fourier Transform (FrFT) domain the energy of chirp signals can concentrate on a specific transform order, and at the same time, the energy of sinusoidal signals may spread over the whole FrFT domain. Hence, these two types of signals can be easily separated through properly designed filter in the FrFT domain, and very limited interference will be generated from each other [23-24]. However, since chirp signal and sinusoidal signal shows energy concentration property in FrFT domain and Frequency domain, respectively, they will show similar property if only one type of signal, i.e., chirp signal or sinusoidal signal is used. Hence, in communication systems, chirp signal and FrFT are usually used in the scenario that two types of signals are used [25].

Fractional Fourier transform-chirp signal processing also has its limitations. For example, the FRFT has one major drawback due to using global kernel, i.e., it only provides such fractional spectral content with no indication about the time localization of the fractional spectral components. However, such limitation could be solved by recent advances in [26] and [27].

Through the abovementioned analysis, we can use sinusoidal signal and chirp signal for primary user and secondary user, respectively. Fractional Fourier transform domain processing will be used in the receiver end so that the interference between primary users and secondary users can be significantly reduced in cooperative spectrum sharing. Neither reducing the transmission data rate nor increasing the realization complexity, Fractional Fourier transform domain signal processing method could bring us an original horizon to handle interference problem.

The contribution of our work can be summarized as:

(1) A novel two-time slot cooperative spectrum sharing protocol employing FrFT domain signal processing technique as well as an adaptive relaying mechanism is proposed.

(2) An optimized power allocation algorithm based on statistical channel gain is proposed and we derived the asymptotic lower bound for the power allocation parameter and obtain the sub-optimal value through Newton iteration method.

(3) We also propose a relay selection algorithm considering the channel status of three links, i.e., PT-ST link, ST-SR link, and ST-PR link, to further improve the performance of secondary system.

The remainder of this paper is organized as follows: Section II briefly introduces the theory of Fractional Fourier Transform. Section III describes the system model as well as the detailed mechanism of our proposed cooperative spectrum sharing protocol. The analysis on the power allocation and secondary user selection of this proposed protocol is given in Section IV. In Section $\mathrm{V}$, the performance comparison between the proposed cooperative spectrum sharing protocol and the protocol in [8] and [10] is performed. Finally, conclusions are drawn in Section VI.

\section{FRACTIONAL FOURIER TRANSFORM}

Before we started to introduce our proposed content, it is necessary to state all the deployed symbols in whole paper. The name of all symbols and their definitions are listed in Table I.

As an extension of Fourier transform, Fractional Fourier Transform has been introduced to signal processing and wireless communication fields in recent years. Similar to sinusoidal signals being the orthogonal basis function of Fourier transform, chirp signals are the orthogonal basis functions of Fractional Fourier Transform. Hence, like sinusoidal signals in the Fourier domain, chirp signals exhibit a good energy concentration property in the FrFT domain, whereas sinusoidal signals typically exhibit a flat energy distribution in some specific FrFT domain. 
Table I Deployed Symbols and Corresponding Definitions

\begin{tabular}{|c|c|}
\hline $\begin{array}{l}\text { Deployed } \\
\text { Symbols }\end{array}$ & Definition \\
\hline$\alpha$ & Transform order of FrFT \\
\hline$\beta$ & Amplification factor in AF relaying mechanism \\
\hline$e$ & Signal separation error \\
\hline$\rho$ & Guaranteed SNR threshold of the primary user \\
\hline$\xi$ & Loss coefficient of the power amplifier \\
\hline$h_{x y}$ & Channel coefficient of the link between terminal $x$ and $y$ \\
\hline$g_{x y}$ & $\begin{array}{c}\text { Rayleigh distributed fading coefficient between terminal } x \text { and } \\
y\end{array}$ \\
\hline$d_{x y}$ & Normalized distance between terminal $x$ and $y$ \\
\hline$d_{1}$ & Radius of outer circle \\
\hline$d_{2}$ & Radius of inner circle \\
\hline$v$ & Path loss exponent \\
\hline$x_{p}$ & Transmit signals of the primary user \\
\hline$x_{s}$ & Transmit signals of the secondary user \\
\hline $\begin{array}{c}x_{s} \text { filter } \\
w_{i j}\end{array}$ & $\begin{array}{c}\text { Filtered out transmit secondary signals after FrFT } \\
\text { Additive white Gaussian noise }\end{array}$ \\
\hline$\sigma_{w}^{2}$ & Power of additive Gaussian white noise \\
\hline$\gamma_{13}$ & Signal to noise ratio of $\mathrm{PT} \rightarrow \mathrm{ST}$ link \\
\hline$O_{p}$ & Outage probability of the primary transmission \\
\hline$O_{s}$ & Outage probability of the secondary transmission \\
\hline$O_{p_{-} \operatorname{sim}}$ & Simplified outage probability of the primary user \\
\hline$O_{\max }$ & Maximum allowable outage probability of the primary user \\
\hline$R_{p}^{A F}$ & Achievable data rate at PR with AF mechanism \\
\hline$R_{p}^{D F}$ & Achievable data rate at PR with DF mechanism \\
\hline$R_{p}$ & Achievable data rate at $\mathrm{PR}$ with $\mathrm{AF} / \mathrm{DF}$ mechanism \\
\hline$R_{s}$ & Achievable data rate of SR \\
\hline$R_{p t}$ & Target data rate of the primary user \\
\hline$R_{s t}$ & Target data rate of the secondary user \\
\hline$P_{r}$ & Transmit power for relaying primary signals at ST \\
\hline$P_{a}$ & Transmit power for sending secondary signals at ST \\
\hline$P_{s}$ & Total transmit power of each secondary user \\
\hline$P_{s_{-} \text {threshold }}$ & $\begin{array}{l}\text { Given threshold of maximum transmit power of the secondary } \\
\text { user }\end{array}$ \\
\hline$P_{p}$ & Total transmit power of primary user \\
\hline$P_{c s}$ & Circuit power consumption of secondary system \\
\hline$P_{c p}$ & Circuit power consumption of primary system \\
\hline$\eta$ & Power allocation parameter \\
\hline$\eta^{*}$ & Optimal power allocation parameter \\
\hline$\eta_{a}$ & Approximate solution of optimal power allocation parameter \\
\hline$\varepsilon_{s}^{E E}$ & Average energy efficiency of secondary system \\
\hline$\varepsilon_{\text {whole }}^{E E}$ & Average energy efficiency of whole system \\
\hline$N$ & Number of available secondary users \\
\hline$M_{i}$ & Priority of each secondary user \\
\hline
\end{tabular}

According to the definition of Fractional Fourier Transform, the FrFT of any signal $f(t)$ can be expressed as follows [28]:

$$
X_{\alpha}(u)=\int_{-\infty}^{+\infty} f(t) K_{\alpha}(u, t) d t,
$$

where, $\alpha$ is the transform order, $K_{\alpha}(u, t)$ is the kernel function of FrFT which is denoted as:

$$
K_{\alpha}(u, t)=\sqrt{\frac{1-j \cot (\alpha)}{2 \pi}} \exp \left(j\left(\frac{t^{2}+u^{2}}{2} \cot (\alpha)-u t \csc (\alpha)\right)\right) \text {. }
$$

Specially, when $\alpha=\pi / 2$, (1) can be simplified as $X_{\pi / 2}(u)=\int_{-\infty}^{+\infty} f(t) \exp (-j 2 \pi u t) d t$, which turns out to be the Fourier transform of signal $f(t)$.

The sinusoidal signal and chirp signal in the time domain are defined as:

$$
\begin{gathered}
s(t)=A \exp \left(j\left(2 \pi f_{0} t+\varphi_{0}\right)\right), \\
c(t)=A \exp \left(j\left(2 \pi f_{0} t+\varphi_{0}+k \pi t^{2}\right)\right), \quad|t| \leq T / 2,
\end{gathered}
$$

where, $A$ is the amplitude, $f_{0}$ is the center frequency; $\varphi_{0}$ is the initial phase; $k$ is the chirp rate and $T$ is the signal duration.

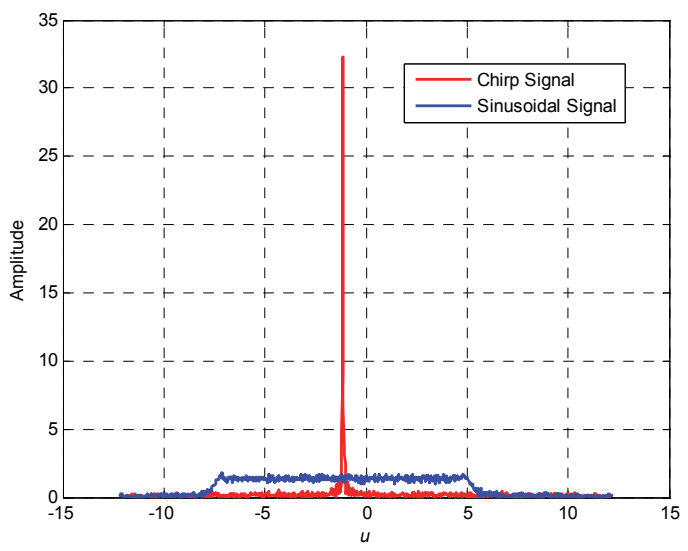

Fig. 1. Sinusoidal signal and chirp signal in the FrFT domain

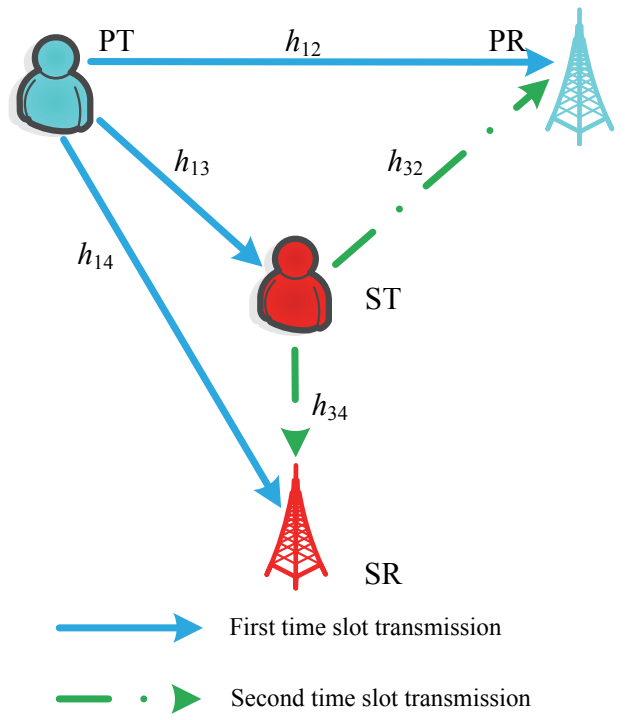

Fig. 2. System model with single secondary user

In order to simplify derivations, we assume $f_{0}=0$ and $\varphi_{0}=0$. The sinusoidal signal $s(t)$ and chirp signal $c(t)$ in the FrFT domain can be expressed as:

$$
\begin{gathered}
S_{\alpha}(u)=\sqrt{1+j \tan (\alpha)} \exp \left(-j \frac{u^{2}}{2} \tan (\alpha)\right), \\
C_{\alpha}(u)=\left\{\begin{array}{l}
\delta(u), \quad \alpha-\arctan (k)-\frac{\pi}{2}=l \pi \\
\sqrt{\frac{1+j \tan (\alpha)}{1+k \tan (\alpha)}} \exp \left(j \frac{u^{2}}{2}\left(\frac{k-\tan (\alpha)}{1+k \tan (\alpha)}\right)\right), \text { others }
\end{array}\right.
\end{gathered}
$$

From (5) and (6), when $\alpha=\arctan (k)+(1 / 2+l) \pi$, we could find that the chirp signal has a peak in the corresponding FrFT domain while the energy of sinusoidal signal is spread over the whole FrFT domain. Hence, by selecting an appropriate transform order $\alpha$, the properly designed chirp signal will show the best energy concentration property and the sinusoidal signal will show a flat energy distribution in the FrFT domain as shown in Fig.1. So these two types of signals can be easily separated through filtering in the Fractional Fourier domain [29]. 


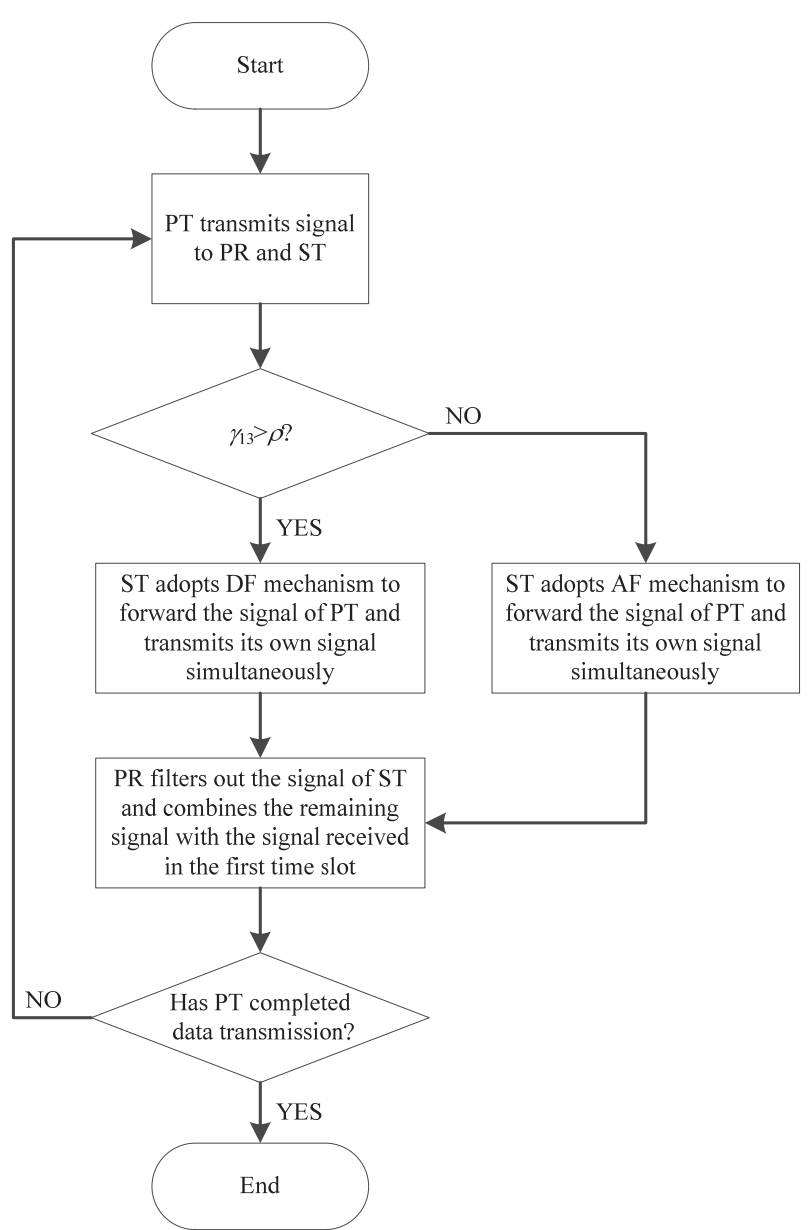

Fig. 3. The transmission procedure for our proposed protocol

In order to quantify the separation property of these two waveforms, the signal separation error $e$ is defined as the ratio of the average amplitude of remaining interference chirp signal to that of the original chirp signal after the filtering.

In this paper, $e$ is restricted to be smaller than 0.1 according to [29]. Considering the above characteristic, in our proposed protocol we use sinusoidal signal and chirp signal for primary system and secondary system, respectively.

\section{System Model AND PROTOCOL DESCRIPTION}

\section{Spectrum Sharing System Model}

In this Section, an overlay cognitive radio network with single secondary user is considered. As shown in Fig.2, the primary system consists of a primary transmitter PT and a primary receiver $\mathrm{PR}$, respectively. The secondary system also contains a pair of transmitter ST and receiver SR. Assuming that the channels over all links are independent and the channel coefficient of the link between terminal $x$ and terminal $y$ is denoted as $h_{x y}=\sqrt{d_{x y}^{-v}} g_{x y}$ where $v$ is the path loss exponent, $d_{x y}$ is the normalized distance and $g_{x y}$ represents the fading coefficient which is Rayleigh distributed, then $\left|h_{x y}\right|^{2}$ is exponentially distributed with mean $1 / \lambda_{x y}$ and $\lambda_{x y}=d_{x y}^{v}$ [8].

Besides, the primary and secondary nodes are equipped with single antenna and operate in a fixed TDMA mode. The signals transmitted by the primary user and secondary user are expressed as $x_{p}$ and $x_{s}$, respectively, with the assumption of $E\left\{\left|x_{p}\right|^{2}\right\}=E\left\{\left|x_{s}\right|^{2}\right\}=1$.

\section{The Proposed Spectrum Sharing Protocol}

In this paper, a two-time slot spectrum sharing protocol using transform domain processing based adaptive relaying is considered. The transmission procedure for this protocol is given in Fig. 3 and explained by the following phase. Here, we only consider the scenario that the primary user needs the help of secondary user, i.e., the spectrum sharing protocol will be used.

In the first time slot, the signal $x^{(1)}$ from PT is transmitted and $x^{(1)}=x_{p}$. The signals received by PR and ST are denoted as $y_{12}$ and $y_{13}$, respectively. Then, we have

$$
\begin{aligned}
& y_{12}=\sqrt{P_{p}} h_{12} x_{p}+w_{12}, \\
& y_{13}=\sqrt{P_{p}} h_{13} x_{p}+w_{13},
\end{aligned}
$$

where, $P_{p}$ is the transmit power of PT and $w_{i j}$ represents the Gaussian noise with the power of $\sigma_{w}^{2}$. In our paper, we assume that the transmission bandwidth is normalized to $1 \mathrm{~Hz}$. Thus, the achievable data rate of PR and ST can be expressed as $R_{12}$ and $R_{13}$, respectively.

$$
\begin{aligned}
& R_{12}=\frac{1}{2} \log _{2}\left(1+\frac{P_{p}\left|h_{12}\right|^{2}}{\sigma_{w}^{2}}\right), \\
& R_{13}=\frac{1}{2} \log _{2}\left(1+\frac{P_{p}\left|h_{13}\right|^{2}}{\sigma_{w}^{2}}\right),
\end{aligned}
$$

where, the factor $1 / 2$ is owing to the fact that the complete transmission process is divided into two time slots. The Signal to Noise Ratio (SNR) $\gamma_{13}$ of PT $\rightarrow$ ST link can be expressed as $\gamma_{13}=\left(P_{p}\left|h_{13}\right|^{2}\right) / \sigma_{w}^{2}$.

In the second time slot, ST adopts adaptive relaying mechanism to forward the signal of primary user. To be specific, ST adaptively chooses DF or AF mechanism depending on whether $\gamma_{13}$ is higher than a given threshold $\rho=2^{2 R_{p t}}-1$, where $R_{p t}$ is the target data rate of primary user, and $\gamma_{13}$ denotes the SNR of PT $\rightarrow$ ST link.

When $\gamma_{13} \geq \rho$, ST can successfully decode the primary signal $x_{p}$ from the received signal $y_{13}$ and DF mechanism is adopted to relay the primary signal. Then the transmitted signal from ST $x^{(2)}$ can be denoted as the linear superposition of the regenerated signal $x_{p}$ with power $P_{r}$ and its own information signal $x_{s}$ with power $P_{a}$, i.e., $x^{(2)}=\sqrt{P_{r}} x_{p}+\sqrt{P_{a}} x_{s}$. Then at $\mathrm{PR}$ and SR, the received signals in the second time slot are:

$$
\begin{aligned}
& y_{32}^{D F}=\sqrt{P_{r}} h_{32} x_{p}+\sqrt{P_{a}} h_{32} x_{s}+w_{32}, \\
& y_{34}^{D F}=\sqrt{P_{r}} h_{34} x_{p}+\sqrt{P_{a}} h_{34} x_{s}+w_{34} .
\end{aligned}
$$

If $r_{13}<\rho$, ST cannot decode the primary signal $x_{p}$, and AF mechanism will be utilized. In this way, the transmitted signal $x^{(2)}$ from ST is the sum of the amplified received signal and its own information signal, i.e., $x^{(2)}=\sqrt{\beta} \sqrt{P_{p}} y_{13}+\sqrt{P_{a}} x_{s}$, where $\beta$ is the amplification factor. When the power allocated for forwarding the primary signal is $P_{r}$, the value of $\beta$ can be obtained by $\beta=P_{r} /\left(P_{p}\left|h_{13}\right|^{2}+\sigma_{w}^{2}\right)$. The received signals at PR and SR in the second time slot can be written as: 


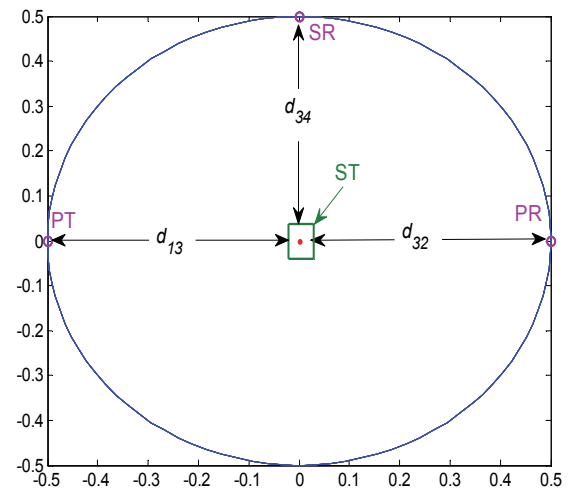

Fig. 4. The location of primary and secondary systems

$$
\begin{aligned}
& y_{32}^{A F}=\sqrt{\beta} \sqrt{P_{p}} h_{13} h_{32} x_{p}+\sqrt{P_{a}} h_{32} x_{s}+\sqrt{\beta} h_{32} w_{13}+w_{32}, \\
& y_{34}^{A F}=\sqrt{\beta} \sqrt{P_{p}} h_{13} h_{34} x_{p}+\sqrt{P_{a}} h_{34} x_{s}+\sqrt{\beta} h_{34} w_{13}+w_{34} .
\end{aligned}
$$

Then signals $y_{12}$ and $y_{32}$ are combined at PR using Maximal Ratio Combination (MRC) to decode $x_{p}$. To improve the decoding performance of primary system, the signal of secondary user $x_{s}$ should be filtered out from $y_{32}^{D F}$ or $y_{32}^{A F}$ using the energy concentration property of chirp signals in the FrFT domain. After removing the estimation of $x_{s}$ from the received signal with the assumption of perfect channel estimation, the remaining signal with different relaying mechanism can be denoted as:

$$
\begin{gathered}
y_{p m}^{D F}=\sqrt{P_{r}} h_{32} x_{p}+\sqrt{P_{a}} h_{32}\left(x_{s}-x_{s_{-} \text {filter }}\right)+w_{32}, \\
y_{p m}^{A F}=\sqrt{\beta} \sqrt{P_{p}} h_{13} h_{32} x_{p}+\sqrt{P_{a}} h_{32}\left(x_{s}-x_{s_{-} \text {filter }}\right)+\sqrt{\beta} h_{32} w_{13}+w_{32},
\end{gathered}
$$

where, $x_{s}$ filter represents the filtered out signal and can be expressed as $x_{s_{-} \text {filter }}=(1-e) x_{s}$. Then, combining the received signals $y_{12}$ and $y_{p m}^{D F}\left(y_{p m}^{A F}\right)$ at PR, the achievable data rate of PR with DF and AF mechanism can be computed by (17) and (18), respectively.

$$
\begin{gathered}
R_{p}^{D F}=\frac{1}{2} \log _{2}\left(1+\frac{P_{p}\left|h_{12}\right|^{2}}{\sigma_{w}^{2}}+\frac{P_{r}\left|h_{32}\right|^{2}}{e^{2} P_{a}\left|h_{32}\right|^{2}+\sigma_{w}^{2}}\right), \\
R_{p}^{A F}=\frac{1}{2} \log _{2}\left(1+\frac{P_{p}\left|h_{12}\right|^{2}}{\sigma_{w}^{2}}+\frac{P_{p}\left|h_{13}\right|^{2} \beta\left|h_{32}\right|^{2}}{e^{2} P_{a}\left|h_{32}\right|^{2}+\sigma_{w}^{2} \beta\left|h_{32}\right|^{2}+\sigma_{w}^{2}}\right) .
\end{gathered}
$$

At SR, we can utilize Fractional Fourier domain filtering to directly decode and obtain the secondary user's signal $x_{s}$ instead of subtracting the interference signal $x_{p}$. The filtered out signal is irrelevant to the forwarding mode adopted by ST and is shown in (19) with perfect channel estimation.

$$
y_{s m}=\sqrt{P_{a}} h_{32} x_{s_{-} \text {filter }}+w_{32} .
$$

Then, the achievable rate of SR can be expressed as:

$$
R_{s}=\frac{1}{2} \log _{2}\left(1+\frac{(1-e)^{2} P_{a}\left|h_{34}\right|^{2}}{\sigma_{w}^{2}}\right) .
$$

In our proposed cooperative spectrum sharing protocol, secondary signals can be easily separated from primary signals through Fractional Fourier domain filtering thanks to the energy concentration property of chirp signals in the FrFT domain and the interference to primary system can be greatly reduced. Moreover, the adaptive relaying mechanism applied to cooperation makes our proposed protocol more flexible and suitable for different channel conditions. Secondary users can also get more opportunities to access the spectrum. Thus, spectrum utilization can be effectively improved and a better communication performance of both the primary and secondary systems can be obtained.

\section{Optimal Power Allocation AND Secondary USER SELECTION}

\section{Power Allocation Scheme}

In order to achieve the optimal performance of the proposed spectrum sharing protocol, we need to analyze the influence of power allocation to the performance of primary user and secondary user and find the optimal power allocation in this Section.

Current power allocation schemes in spectrum sharing protocols can be classified into two types: one is based on instantaneous channel condition and the other one is according to statistical channel condition. Due to the time-varying characteristic of wireless channel, it will cost a lot of signaling overhead to obtain the instantaneous channel gain in a real system. However, the statistics of the channel gain remain relatively stable and are only related to some system parameters, such as transmission distance and wavelength of the electromagnetic. Thus the statistics of channel gain can be more easily obtained and we use the statistics of channel gain to perform optimal power allocation in our proposed protocol.

Before the relay selection and power allocation process, we could obtain these statistics channel gains by long term statistic estimation. Then, the selected ST could act as the relay for a period of time and obtaining signaling of the instantaneous channel gain during each transmission time slot is unnecessary.

Thus, to reduce the signaling overhead of power allocation and relay selection process, we use outage probability based on statistics as the metric to evaluate the performance of power allocation optimization and relay selection process.

The definition of outage probability is the probability that the instantaneous achievable data rate is below the target data rate [30]. Then the outage probability of the primary transmission in our proposed protocol is given by

$$
O_{p}=\operatorname{Pr}\left\{R_{13} \geq R_{p t}\right\} \operatorname{Pr}\left\{R_{p}^{D F}<R_{p t}\right\}+\operatorname{Pr}\left\{R_{13}<R_{p t}\right\} \operatorname{Pr}\left\{R_{p}^{A F}<R_{p t}\right\}
$$

where, $R_{13}$ denotes the transmission data rate at ST. $R_{p}^{A F}$ and $R_{p}^{D F}$ represents the achievable data rate at $\mathrm{PR}$ with $\mathrm{DF}$ and $\mathrm{AF}$ mechanism, respectively. $R_{p t}$ is the target data rate of primary user, which could be expressed as $(1 / 2) \log _{2}(1+\rho)$, where $\rho$ is the SNR threshold.

The outage probability of secondary signal transmission with the secondary user target data rate $R_{s t}$ is given by

$$
O_{s}=\operatorname{Pr}\left\{R_{s}<R_{s t}\right\} \text {. }
$$

Since the secondary system should not introduce excessive interference to degrade the performance of the primary system significantly, the maximum transmit power of the secondary user is restricted to a given threshold $P_{s_{-} \text {threshold }}$. We assume that $P_{r}+P_{a}=P_{s}$, where $P_{s}$ is the actual transmit power of each secondary user, which is always less than or equal to $P_{s_{-} \text {threshold }}$, 


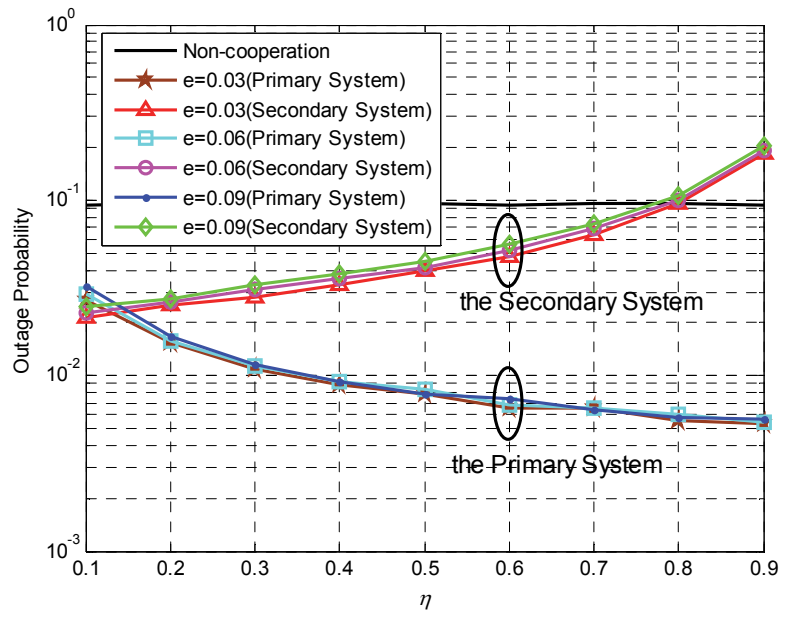

Fig. 5. Outage probability with different $\eta$ and $e$

i.e., $P_{s \text { threshold }} \geq P_{s}$ and then the effect of power allocation parameter $\eta$, where $\eta=P_{r} / P_{s}$, on the performance of outage probability of both primary and secondary systems is analyzed in detail as follows.

The distance between PT and PR is normalized to " 1 ", i.e. $d_{12}=1$ according to [31]. Fig. 4 assumes that the secondary user is located at the middle of PT and PR, i.e. $d_{13}=d_{32}=1 / 2 d_{12}=0.5$, and $d_{34}=0.5 d_{12}=0.5$. The path loss exponent $v=4$, and signal separation error $e=0.1$ and $R_{p t}=R_{s t}=1 \mathrm{bits} / \mathrm{s} / \mathrm{Hz}$. Fig. 5 shows the outage probability of the proposed spectrum sharing protocol with different $\eta$ and $e$. From Fig. 5, we can easily see that with the increase of $\eta$, the outage probability of the primary system decreases while the outage probability of the secondary system increases. The triangle line and the five-pointed star line illustrates that the outage probability of secondary user and primary user when signal separation parameter set as 0.03 , respectively. It is obvious that both primary and secondary user's outage probability is just a bit lower than that of $e=0.06$. Considering the case of $e=0.09$, the outage probability of both systems are just a little higher than that of $e=0.06$. In conclusion, the outage probability of primary user and secondary user is almost the same when the values of $e$ vary. Thus, we let $e$ fix on 0.06 for the following part of our manuscript. Hence, the optimal power allocation parameter needs to be determined in order to minimize the secondary transmission outage probability subject to the outage probability constraint at PR. This optimization problem can be formulated as: $\min O_{s}$,

subject to

$$
\begin{gathered}
O_{p} \leq O_{\max }, \\
P_{r}+P_{a}=P_{s}, \\
P_{r} \geq 0, \\
P_{a} \geq 0, \\
P_{s} \leq P_{s_{-} \text {threshold }} .
\end{gathered}
$$

where, $P_{s_{-} \text {threshold }}$ is the maximum transmit power constraint of secondary user, and $O_{\max }$ is the outage probability constraint of the primary system and we need to find the optimal power

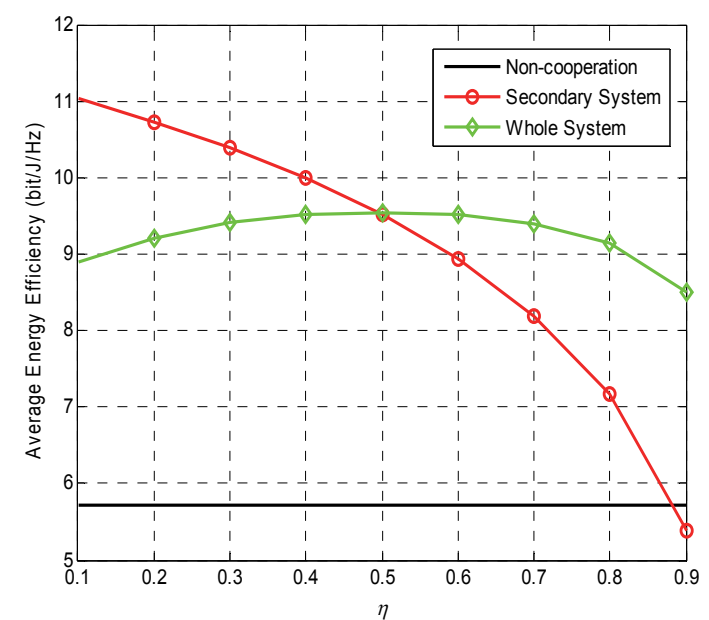

Fig. 6. Average Energy Efficiency versus power allocation parameter $\eta$ allocation parameter $\eta^{*}$ to minimize the outage probability of secondary system $O_{s}$. From the above analysis, it is seen that $O_{s}(\eta)$ is an increasing function of $\eta$ and $O_{p}(\eta)$ is a decreasing function of $\eta$ for $\eta \in[0,1]$. Therefore, the optimal solution $\eta^{*}$ of the optimization problem (23) should satisfy $O_{p}\left(\eta^{*}\right)=O_{\max }$. However, $O_{p}$ in (23) contains several random variables. So a direct solution entails high complexity and the optimal solution $\eta^{*}$ is difficult to obtain. Hence, some reasonable assumptions are made to simplify $O_{p}$ to reduce complexity and obtain an approximate solution $\eta_{a}$ for $\eta_{a} \in\left[\eta^{*}, 1\right]$. In this way, constraint condition (23.b) can be satisfied.

According to (21), the expression of $O_{p}$ includes 4 terms, i.e., $\operatorname{Pr}\left\{R_{13} \geq R_{p t}\right\}, \operatorname{Pr}\left\{R_{13}<R_{p t}\right\}, \operatorname{Pr}\left\{R_{p}^{D F}<R_{p t}\right\}$ and $\operatorname{Pr}\left\{R_{p}^{A F}<R_{p t}\right\}$. Due to the exponent distribution of variable $\left|h_{x y}\right|^{2}$, we firstly simplify the expression of $\operatorname{Pr}\left\{R_{13} \geq R_{p t}\right\}$ and $\operatorname{Pr}\left\{R_{13}<R_{p t}\right\}$. Substituting (10) into $\operatorname{Pr}\left\{R_{13}<R_{p t}\right\}$ in (21), we have

$$
\begin{aligned}
& \operatorname{Pr}\left\{R_{13}<R_{p t}\right\}=\operatorname{Pr}\left\{\frac{1}{2} \log _{2}\left(1+\frac{P_{p}\left|h_{13}\right|^{2}}{\sigma_{w}^{2}}\right)<R_{p t}\right\} \\
& =\operatorname{Pr}\left\{\left|h_{13}\right|^{2}<\frac{\sigma_{w}^{2}}{P_{p}} \rho\right\}=1-\exp \left(-\lambda_{0} \rho\right)
\end{aligned}
$$

where, $\lambda_{0}=d_{13}^{v} \sigma_{w}^{2} / P_{p}$. According to the probability theory, we have

$$
\operatorname{Pr}\left\{R_{13} \geq R_{p t}\right\}=\exp \left(-\lambda_{0} \rho\right) .
$$

Substituting (17) into $\operatorname{Pr}\left\{R_{p}^{D F}<R_{p t}\right\}$ and $\operatorname{Pr}\left\{R_{p}^{D F}<R_{p t}\right\}$ can be written as (26) at the top of next page. Substituting $\beta=P_{r} /\left(P_{p}\left|h_{13}\right|^{2}+\sigma_{w}^{2}\right)$ and (18) into $\operatorname{Pr}\left\{R_{p}^{A F}<R_{p t}\right\}$ in (21), $\operatorname{Pr}\left\{R_{p}^{A}<R_{p t}\right\}$ can be rewritten as (27) at the top of next page.

Before we start to solve the optimization problem in (23), it is crucial for us to evaluate the energy efficiency performances. According to the constraint stated in (23.b), our power allocation and relay selection process is under the premise of guaranteeing the quality of service (QoS) of the primary user. Hence, we do not analyze the energy efficiency of the primary system. We introduce two metrics, i.e., the average energy efficiency of the secondary system and the whole system, to evaluate system performance. 


$$
\begin{gathered}
\operatorname{Pr}\left\{R_{p}^{D F}<R_{p t}\right\}=\operatorname{Pr}\left\{\frac{1}{2} \log _{2}\left(1+\frac{P_{p}\left|h_{12}\right|^{2}}{\sigma_{w}^{2}}+\frac{\eta P_{s}\left|h_{32}\right|^{2}}{e^{2}(1-\eta) P_{s}\left|h_{32}\right|^{2}+\sigma_{w}^{2}}\right)<R_{p t}\right\} \\
\operatorname{Pr}\left\{R_{p}^{A F}<R_{p t}\right\}=\operatorname{Pr}\left\{\frac{1}{2} \log _{2}\left(1+\frac{P_{p}\left|h_{12}\right|^{2}}{\sigma_{w}^{2}}+\frac{\eta P_{p}\left|h_{13}\right|^{2} P_{s}\left|h_{32}\right|^{2}}{e^{2}(1-\eta) P_{s}\left|h_{32}\right|^{2}\left(P_{p}\left|h_{13}\right|^{2}+\sigma_{w}^{2}\right)+\eta P_{s}\left|h_{32}\right|^{2} \sigma_{w}^{2}+\left(P_{p}\left|h_{13}\right|^{2}+\sigma_{w}^{2}\right) \sigma_{w}^{2}}\right)<R_{p t}\right\}
\end{gathered}
$$

According to [32], the expressions of these two metrics are shown in (28) and (29), respectively.

$$
\begin{gathered}
\varepsilon_{s}^{E E}=\frac{R_{s}}{\frac{1}{2} \xi \cdot P_{s}+P_{c s}}, \\
\varepsilon_{\text {whole }}^{E E}=\frac{R_{s}+R_{p}}{\frac{1}{2}\left(\xi \cdot P_{s}+\xi \cdot P_{p}\right)+P_{c s}+P_{c p}} .
\end{gathered}
$$

where, $\xi$ represents the amplifier efficiency of the power amplifier and $R_{p}$ is the achievable data rate at PR with either DF or AF mechanism. $P_{c s}$ and $P_{c p}$ are the circuit power consumption of secondary system and primary system, respectively. The factor $1 / 2$ in (28) and (29) accounts for the fact that the whole transmission process is divided into two equal sub-time slots.

Fig. 6 provides the average energy efficiency of the secondary system and the whole system versus the power allocation parameter $\eta$. From Fig. 6, when $\eta$ increases, the average energy efficiency of the secondary system decreases and the average energy efficiency of the whole two systems increases at first, and then it decreases. When $\eta$ is small, the average channel quality of the primary user link, i.e., the PT-ST link and the ST-PR link, must be good enough. Then, the secondary user will use most of its remaining power to transmit its own signal, and hence the secondary user will have higher average energy efficiency. Along with the increase in the value of $\eta$, the remaining power for the secondary user decreases, and thus, the average energy efficiency decreases, too.

The average energy efficiency of the whole system combines both the primary system and the secondary system. When $\eta$ approaches 1, the average energy efficiency of these two systems have the opposite behavior. The highest average energy efficiency of the whole system occurs in the situation where $\eta=0.54$. However, we can also find that $\eta$ has limited influence on the average energy efficiency performance of the whole system, and hence, in the next Sub-Section, we will explain the specific steps to obtain the sub-optimal value of $\eta_{\mathrm{a}}$ based on the objective of minimizing the outage probability of the secondary transmission $O_{s}$.

\section{The Sub-Optimal Solution for Power Allocation parameter}

Due to the expression of $\operatorname{Pr}\left\{R_{p}^{D F}<R_{p t}\right\}$ and $\operatorname{Pr}\left\{R_{p}^{A F}<R_{p t}\right\}$ is complex and entails multiple variables in one terms, we need to make some assumptions to reduce the calculation complexity. To solve the optimization problem and at the same time satisfy this constraint, we should restrictively guarantee that $O_{p}$ is always less than $O_{\max }$. We need to amplify the exact value of $O_{p}$ and then obtain the upper bound of $O_{p}$. Thus, we make several assumptions below to realize it through two ways. One is to amplify the value of $\operatorname{Pr}\left\{R_{p}^{D F}<R_{p t}\right\}$ and the
other one is to amplify the value of $\operatorname{Pr}\left\{R_{p}^{A F}<R_{p t}\right\}$.

Let us begin with the process of amplifying the value of $\operatorname{Pr}\left\{R_{p}^{D F}<R_{p t}\right\} . h_{32}$ is the channel gain of the ST-PR link. Since $\left|h_{32}\right|^{2}$ obeys the exponential distribution, the mean value of this variable could be expressed as $1 / \lambda_{32}$. According to [8], the mean value of $\left|h_{32}\right|^{2}$ is less than $-7 \mathrm{~dB}$ and the maximum value of $\left|h_{32}\right|^{2}$ is definitely less than $0 \mathrm{~dB}$. The ratio of $P_{s} / \sigma_{w}^{2}$ is set to $10 \mathrm{~dB}$ as in [8], and the maximum value of the signal separation error $e$ is always less than 0.1 according to [29], and hence, the conclusion that $e^{2} P_{s}\left|h_{32}\right|^{2} / \sigma_{w}^{2}$ is less than 1 could be satisfied all the time. Therefore, we make the assumption that $e^{2} P_{s}\left|h_{32}\right|^{2}=\sigma_{w}^{2}$ to amplify the denominator of the third term in (26) in order to simplify the analysis of the term $\operatorname{Pr}\left\{R_{p}^{D F}<R_{p t}\right\}$. Based on our assumptions, $\operatorname{Pr}\left\{R_{p}^{D F}<R_{p t}\right\}$ can therefore be denoted as (30).

$$
\operatorname{Pr}\left\{R_{p}^{D F}<R_{p t}\right\}=\operatorname{Pr}\left\{\frac{1}{2} \log _{2}\left(1+\frac{P_{p}\left|h_{12}\right|^{2}}{\sigma_{w}^{2}}+\frac{\eta}{2-\eta} \frac{P_{s}\left|h_{32}\right|^{2}}{\sigma_{w}^{2}}\right)<R_{p t}\right\} .
$$

By amplifying the interference and noise, the obtained approximate solution $\eta_{a}$ is a little larger than the optimal solution $\eta^{*}$. Hence, the outage probability of primary user with power allocation parameter $\eta_{a}$ will inevitably satisfy the outage probability constraint. After simplification, we can see that $\operatorname{Pr}\left\{R_{p}^{D F}<R_{p t}\right\}$ is the summation of two exponential random variables. According to cumulative distribution function (CDF) deduced in [33], (31) can be expressed as:

$$
\operatorname{Pr}\left\{R_{p}^{D F}<R_{p t}\right\}=1-\frac{\lambda_{1}}{\lambda_{1}-\lambda_{2}} \exp \left(-\lambda_{2} \rho\right)+\frac{\lambda_{2}}{\lambda_{1}-\lambda_{2}} \exp \left(-\lambda_{1} \rho\right)
$$

where, $\lambda_{1}=d_{12}^{v} \sigma_{w}^{2} / P_{p}, \lambda_{2}=(2-\eta) d_{32}^{v} \sigma_{w}^{2} /\left(\eta P_{s}\right)$.

Similarly, some assumptions need to be made to simplify $\operatorname{Pr}\left\{R_{p}^{A F}<R_{p t}\right\}$. ST adopts AF mechanism which means that $r_{13}<\rho$. So $P_{p}\left|h_{13}\right|^{2}<\rho \sigma_{w}^{2}$ and $\left(P_{p}\left|h_{13}\right|^{2}+\sigma_{w}^{2}\right)<(\rho+1) \sigma_{w}^{2}$ can be obtained. Moreover, since $P_{s}\left|h_{32}\right|^{2}>\sigma_{w}^{2}$, we have $\left(P_{p}\left|h_{13}\right|^{2}+\sigma_{w}^{2}\right)<(\rho+1) P_{s}\left|h_{32}\right|^{2}$.

In order to reduce the computational complexity, we use $\sigma_{w}^{2}$ and $(\rho+1) P_{s}\left|h_{32}\right|^{2}$ to replace $e^{2} P_{s}\left|h_{32}\right|^{2}$ and $P_{p}\left|h_{13}\right|^{2}+\sigma_{w}^{2}{ }^{w}$, respectively, in (27). Then $\operatorname{Pr}\left\{R_{p}^{A F}<R_{p t}\right\}$ can be denoted as:

$$
\begin{aligned}
\operatorname{Pr}\left\{R_{p}^{A F}<R_{p t}\right\} & =\operatorname{Pr}\left\{\left(\frac{P_{p}\left|h_{12}\right|^{2}}{\sigma_{w}^{2}}+\frac{\eta}{2(\rho+1)-\eta \rho} \frac{P_{s}\left|h_{13}\right|^{2}}{\sigma_{w}^{2}}\right)<R_{p t}\right\} \\
& =1-\frac{\lambda_{1}}{\lambda_{1}-\lambda_{3}} \exp \left(-\lambda_{3} \rho\right)+\frac{\lambda_{3}}{\lambda_{1}-\lambda_{3}} \exp \left(-\lambda_{1} \rho\right)
\end{aligned}
$$

where, $\lambda_{3}=(2(\rho+1)-\eta \rho) d_{13}^{v} \sigma_{w}^{2} /\left(\eta P_{s}\right)$.

Through a series of simplification above, $O_{p}$ could be expressed as (33) at the top of next page. To solve our problems easier, it is obvious for us to find that $O_{p}$ and $O_{s}$ are monotonic functions with independent variable $\eta$ shown in Fig. 5. 


$$
\begin{gathered}
O_{p_{-} \text {sim }}=\left(\exp \left(-\lambda_{0} \rho\right)\right)\left(1-\frac{\lambda_{1}}{\lambda_{1}-\lambda_{2}} \exp \left(-\lambda_{2} \rho\right)+\frac{\lambda_{2}}{\lambda_{1}-\lambda_{2}} \exp \left(-\lambda_{1} \rho\right)\right)+\left(1-\exp \left(-\lambda_{0} \rho\right)\right)\left(1-\frac{\lambda_{1}}{\lambda_{1}-\lambda_{3}} \exp \left(-\lambda_{3} \rho\right)+\frac{\lambda_{3}}{\lambda_{1}-\lambda_{3}} \exp \left(-\lambda_{1} \rho\right)\right) \\
\operatorname{Pr}\left\{R_{p}^{A F}<R_{p t}\right\}=\operatorname{Pr}\left\{\frac{\eta\left|h_{13}\right|^{2} P_{s}\left|h_{32}\right|^{2}}{e^{2}(1-\eta) P_{s}\left|h_{32}\right|^{2}\left(\gamma\left|h_{13}\right|^{2}+1\right)+\eta P_{s}\left|h_{32}\right|^{2}+\left(P_{p}\left|h_{13}\right|^{2}+\sigma_{w}^{2}\right)}<\frac{\rho}{\gamma}-\left|h_{12}\right|^{2}\right\}
\end{gathered}
$$

From Fig.5, we can observe that the outage probability of primary system and secondary system decreases and increases with $\eta$ monotonically, respectively. Hence, minimizing $O_{s}$ means minimizing $\eta$ and (23.a) can then be replaced by (34.a). Substituting (32) into (23.b), (23.b) can then be replaced by (34.b). Substituting (23.c) into (23.d), we have $\eta=P_{r} / P_{s}=1-P_{a} / P_{s}$. Then according to (23.d) and (23.e), we can obtain $0 \leq \eta \leq 1$, i.e. (34.c). Thus, the optimization problem described by (23) can be transformed into (34). $\min \eta$,

subject to

$$
\begin{gathered}
O_{p_{-} \text {sim }} \leq O_{\max }, \\
0 \leq \eta \leq 1 .
\end{gathered}
$$

Observing (33) carefully, we could find that the value of $O_{p}$ is determined by two variables, i.e., the distance between PT and ST $d_{13}$ and the power allocation parameter $\eta$. Then, when $d_{13}$ is given, $O_{p_{-} \text {sim }}$ is monotonic with $\eta$. Using iteration method, such as Newton iteration method, is efficient to obtain the zero point of monotonic function in a given interval [34]. Thus, we utilize the Newton iteration method and revised Newton iteration method to solve the equation $O_{p_{\text {sim }}}=O_{\max }$ in (34) within the feasible region of $\eta \in[0,1]$.

\section{Asymptotic analysis for solving power allocation parameter}

We adopt asymptotic analysis method to obtain the lower bound of power allocation parameter $\eta^{*}$. We focused on (23.b) to obtain the lower bound of $O_{p}$ first. Specifically, we can find from (21) that there are 4 terms in the expression of $O_{p}$. Apart from the term $\operatorname{Pr}\left\{R_{p}^{A F}<R_{p t}\right\}$, the other three terms are able to obtain the exact value according to probability theory. The third term in the expression of $O_{p} \operatorname{Pr}\left\{R_{p}^{A F}<R_{p t}\right\}$ is difficult to be solved because it contains two random variables, i.e., $\left|h_{13}\right|^{2}$ and $\left|h_{32}\right|^{2}$. Thus, it is necessary to obtain the lower bound of $\operatorname{Pr}\left\{R_{p}^{A F}<R_{p t}\right\}$ and further calculate the lower bound of $O_{p}$ and then the lower bound of $\eta^{*}$ could be found. Firstly, we could easily transform the expression from (27) to (35) at the top of this page, where $\rho=2^{2 R_{p t}}-1$ and $\gamma=P_{p} / \sigma_{w}^{2}$.

Then, we can use asymptotic analysis to obtain the lower bound of $\operatorname{Pr}\left\{R_{p}^{A F}<R_{p t}\right\}$. Based on the proof of Lemma 1 in [35], we could obtain the lower bound of a probability function containing two random variables by calculating this limit:

$$
\lim _{\delta \rightarrow 0} \operatorname{Pr}\left[r_{\delta}<h(\delta)\right] / h(\delta) \geq \alpha_{1}+\alpha_{2} .
$$

where, $h(\delta)$ is a constant function and $r_{\delta}$ represents the probability function and $\alpha_{1}$ and $\alpha_{2}$ denotes the transformed mean value of corresponding two random variables in the probability function. If we could obtain the exact value of $\alpha_{1}$ and $\alpha_{2}$ and $h(\delta)$, we can obtain the lower bound of $\operatorname{Pr}\left\{R_{p}^{A F}<R_{p t}\right\}$. Therefore, we then try to obtain the exact solution of $\alpha_{1}$ and $\alpha_{2}$ and $h(\delta)$.

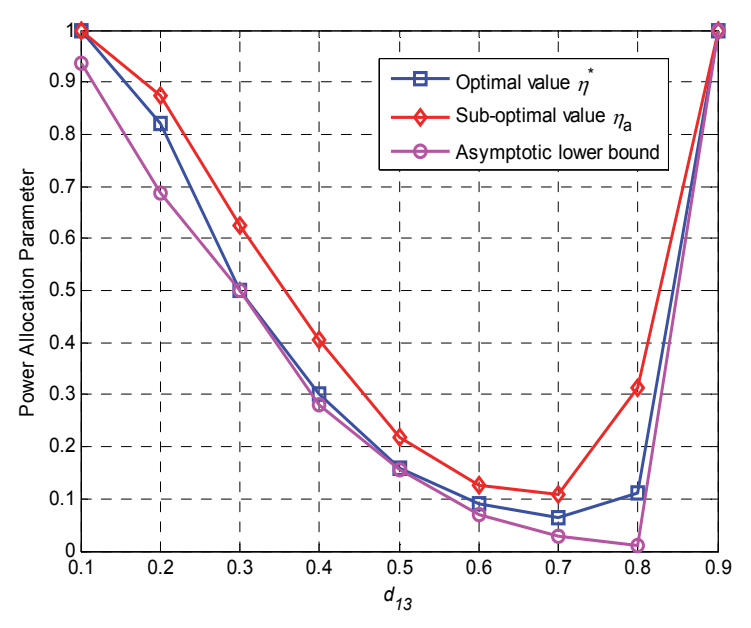

Fig. 7. The lower bound and exact solution for power allocation parameter

Looking at the expression of (35), we define the left part of the inequality in the probability function as $r_{\delta}$. Substituting $r_{\delta}$ into $\operatorname{Pr}\left[r_{\delta}<h(\delta)\right]$ and assuming $h(\delta)$ exists, and then we have:

$$
\begin{aligned}
\operatorname{Pr}\left[r_{\delta}<h(\delta)\right] & =\operatorname{Pr}\left[\frac{\eta\left|h_{13}\right|^{2}\left|h_{32}\right|^{2} P_{s}}{e^{2}(1-\eta) P_{s}\left|h_{32}\right|^{2}\left(\gamma\left|h_{13}\right|^{2}+1\right)+\eta P_{s}\left|h_{32}\right|^{2}+P_{p}\left|h_{13}\right|^{2}+\sigma_{w}^{2}}<h(\delta)\right] \\
& =\operatorname{Pr}\left[\frac{e^{2}(1-\eta) \gamma}{\eta}+\frac{e^{2}(1-\eta)+\eta}{\eta\left|h_{13}\right|^{2}}+\frac{P_{p}}{\eta\left|h_{32}\right|^{2} P_{s}}+\frac{\sigma_{w}^{2}}{\left.\eta\left|h_{13}\right|^{2} h_{32}\right|^{2} P_{s}}>\frac{1}{h(\delta)}\right] . \\
& >\operatorname{Pr}\left[\frac{e^{2}(1-\eta)+\eta}{\eta\left|h_{13}\right|^{2}}+\frac{P_{p}}{\eta\left|h_{32}\right|^{2} P_{s}}>\frac{1}{h(\delta)}\right] \\
& \geq \operatorname{Pr}\left[\max \left(\frac{e^{2}(1-\eta)+\eta}{\eta\left|h_{13}\right|^{2}}, \frac{P_{p}}{\eta\left|h_{32}\right|^{2} P_{s}}\right)>\frac{1}{h(\delta)}\right] \\
& =1-\operatorname{Pr}\left\{\left|h_{13}\right|^{2} \geq\left[e^{2}(1-\eta) / \eta+1\right] h(\delta)\right\} \operatorname{Pr}\left[\left|h_{32}\right|^{2} \geq P_{p} h(\delta) /\left(\eta P_{s}\right)\right]
\end{aligned}
$$

Since $\left|h_{13}\right|^{2}$ and $\left|h_{32}\right|^{2}$ are random variables with exponent distribution, according to the probability theory, we have

$$
\begin{gathered}
\operatorname{Pr}\left\{\left|h_{13}\right|^{2} \geq\left[e^{2}(1-\eta) / \eta+1\right] h(\delta)\right\}=\exp \left[-\alpha_{1} h(\delta)\right] \\
\operatorname{Pr}\left[\left|h_{32}\right|^{2} \geq P_{p} h(\delta) /\left(\eta P_{s}\right)\right]=\exp \left[-\alpha_{2} h(\delta)\right]
\end{gathered}
$$

where, $\alpha_{1}=d_{13}^{v}\left[e^{2}(1-\eta) / \eta+1\right]$ and $\alpha_{2}=d_{32}^{v} P_{p} /\left(\eta P_{s}\right)$, and $\operatorname{Pr}\left[r_{\delta}<h(\delta)\right]^{13}$ could then be cexpressed as $=1-\exp \left[-\left(\alpha_{1}+\alpha_{2}\right) h(\delta)\right]$.

Then, we define $\alpha_{0}$ and $\delta$ as $\alpha_{0}=P_{p}\left|h_{12}\right|^{2} / \sigma_{w}^{2}$ and $\delta=\rho / \gamma$, respectively to obtain $h(\delta)$. According to the property that the random variable $\left|h_{12}\right|^{2}$ follows the exponential distribution, we simplify (35) to obtain $h(\delta)$. Using integration of probability function, we can get (39) at the top of next page.

Note that $\delta$ is a function of transmit SNR $\gamma$ and when $\gamma \rightarrow \infty, \delta \rightarrow 0$ always satisfies. Thus, according to [36], we have: 


$$
\begin{aligned}
\operatorname{Pr}\left\{R_{p}^{A F}<R_{p t}\right\} & =\int_{0}^{\delta} \operatorname{Pr}\left\{\frac{\eta\left|h_{13}\right|^{2} P_{s}\left|h_{32}\right|^{2}}{e^{2}(1-\eta) P_{s}\left|h_{32}\right|^{2}\left(\gamma\left|h_{13}\right|^{2}+1\right)+\eta P_{s}\left|h_{32}\right|^{2}+\left(P_{p}\left|h_{13}\right|^{2}+\sigma_{w}^{2}\right)}<\delta-x\right\} \alpha_{0} \exp \left(-\alpha_{0} x\right) d x \\
& =\int_{0}^{1} \operatorname{Pr}\left\{\frac{\eta\left|h_{13}\right|^{2} P_{s}\left|h_{32}\right|^{2}}{e^{2}(1-\eta) P_{s}\left|h_{32}\right|^{2}\left(\gamma\left|h_{13}\right|^{2}+1\right)+\eta P_{s}\left|h_{32}\right|^{2}+\left(P_{p}\left|h_{13}\right|^{2}+\sigma_{w}^{2}\right)}<\delta x^{\prime}\right\} \alpha_{0} \exp \left[-\alpha_{0} \delta\left(1-x^{\prime}\right)\right] d x^{\prime} \\
& =\delta^{2} \alpha_{0} \int_{0}^{1} \operatorname{Pr}\left\{\frac{\eta\left|h_{13}\right|^{2} P_{s}\left|h_{32}\right|^{2}}{e^{2}(1-\eta) P_{s}\left|h_{32}\right|^{2}\left(\gamma\left|h_{13}\right|^{2}+1\right)+\eta P_{s}\left|h_{32}\right|^{2}+\left(P_{p}\left|h_{13}\right|^{2}+\sigma_{w}^{2}\right)}<\delta x^{\prime}\right\} \mid \delta x^{\prime} \cdot x^{\prime} \exp \left[-\alpha_{0} \delta\left(1-x^{\prime}\right)\right] d x^{\prime}
\end{aligned}
$$

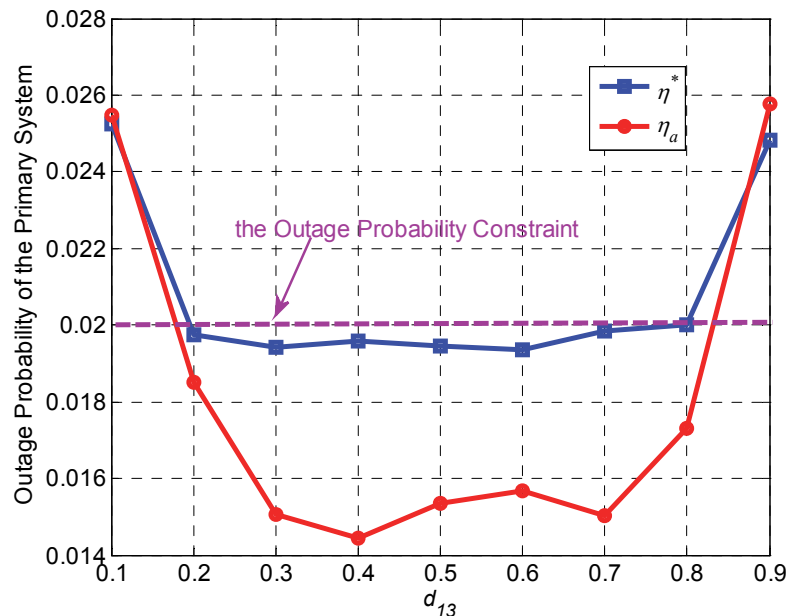

Fig. 8. Outage probability of the primary system

$$
\lim _{\gamma \rightarrow \infty} e^{-\alpha_{0} \delta\left(1-x^{\prime}\right)}=1
$$

Substitute (40) into (39), we can obtain (41) and the lower bound of $\operatorname{Pr}\left\{R_{p}^{A F}<R_{p t}\right\}$ can be calculated when equality of (41) is satisfied:

$$
\lim _{\delta \rightarrow 0} \operatorname{Pr}\left\{R_{p}^{A F}<R_{p t}\right\} / \delta^{2} \geq\left(\alpha_{1}+\alpha_{2}\right) \alpha_{0} / 2
$$

where, $h(\delta)$ equals to $1 / \delta^{2}$. Thus, the lower bound of $\operatorname{Pr}\left\{R_{p}^{A F}<R_{p t}\right\}$ could be expressed by:

$$
\operatorname{Pr}\left\{R_{p}^{A F}<R_{p t}\right\}=\rho \sigma_{w}^{2}\left(\alpha_{1}+\alpha_{2}\right) \alpha_{0} /\left(2 P_{p}\right) .
$$

Substituting (42) and (24) ( 26) into (21), we could obtain the lower bound of $O_{p}$ and $\eta^{*}$. The simulation results of optimal value, sub-optimal value and asymptotic lower bound of power allocation parameter are shown in Fig. 7.

From Fig. 7, we find that the lower bound is almost tight with optimal value of power allocation parameter $\eta^{*}$, and hence the validity of the proposed power allocation algorithm can be verified.

Moreover, the sub-optimal solution of power allocation parameter $\eta_{a}$ is very close to the optimal $\eta^{*}$, which means that the error of using abovementioned procedures is tolerable. However, the complexity and the multiplications using exhaustive method are extremely high. Thus, to obtain the sub-optimal value of power allocation parameter $\eta_{a}$, we use iteration methods, such as the Newton iteration method, and the revised Newton iteration method. Based on these effective iteration methods, the complexity and multiplications could be reduced significantly.

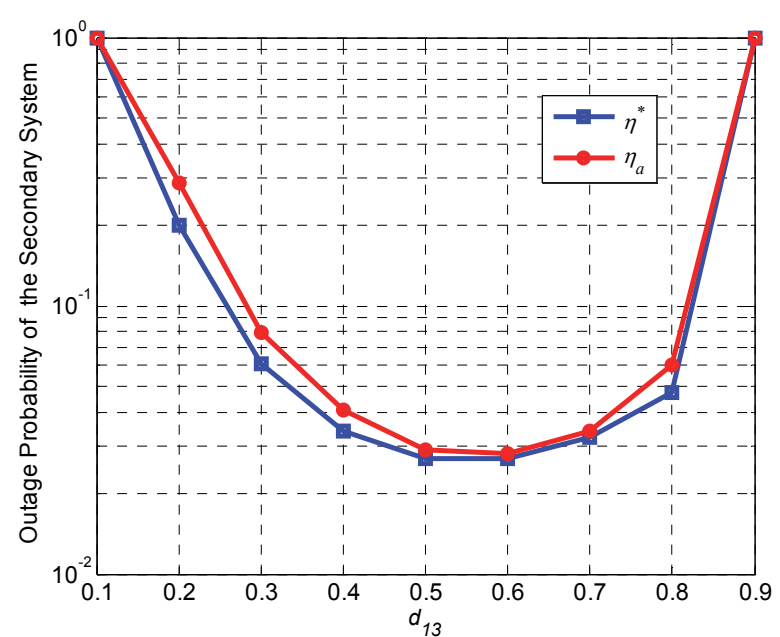

Fig. 9. Outage probability of the secondary system

Furthermore, there is still a gap between $\eta^{*}$ and $\eta_{a}$. The reason for the gap is as follows. When we solve the optimization problem in (34), we have made two assumptions, i.e., $e^{2} P_{s}\left|h_{32}\right|^{2}$ and $P_{p}\left|h_{13}\right|^{2}+\sigma_{w}^{2}$ are replaced by $\sigma_{w}^{2}$ and $(\rho+1) P_{s}\left|h_{32}\right|^{2}$, respectively. In fact, these two assumptions amplify the noise and interference, respectively. In this way, our sub-optimal solution $\eta_{a}$ is a bit higher than $\eta^{*}$. The gap between $\eta^{*}$ and $\eta_{a}$ consists of the amplified interference and noise. When $d_{13}$ locates between 0.5 and 0.7 , the gap is less than 0.04 , and the gap is approximately 0.1 higher than $\eta^{*}$ when $d_{13}$ fixes on other values.

Fig. 8 shows the outage probability of primary system with the optimal and sub-optimal power allocation parameters. From Fig. 8, we can observe that the location of ST is essential for the outage probability performance of primary system. When the distance of PT $\rightarrow$ ST link is either small or large, even if the total power is allocated to assist the primary user, the communication requirement of primary user still cannot be satisfied. In comparison, a secondary user located in a suitable area can assist the primary user through reasonable power allocation algorithm, to reach its target data rate. For instance, using the parameters in Fig. 5, we can find that when $d_{13} \in[0.2,0.8]$, the outage probability constraint of the primary user can be satisfied with both the optimal and sub-optimal solutions $\eta^{*}$ and $\eta_{a}$.

Fig. 9 shows the outage probability of the secondary system with the optimal and sub-optimal power allocation parameters. It can be seen that there is only a very limited difference in 


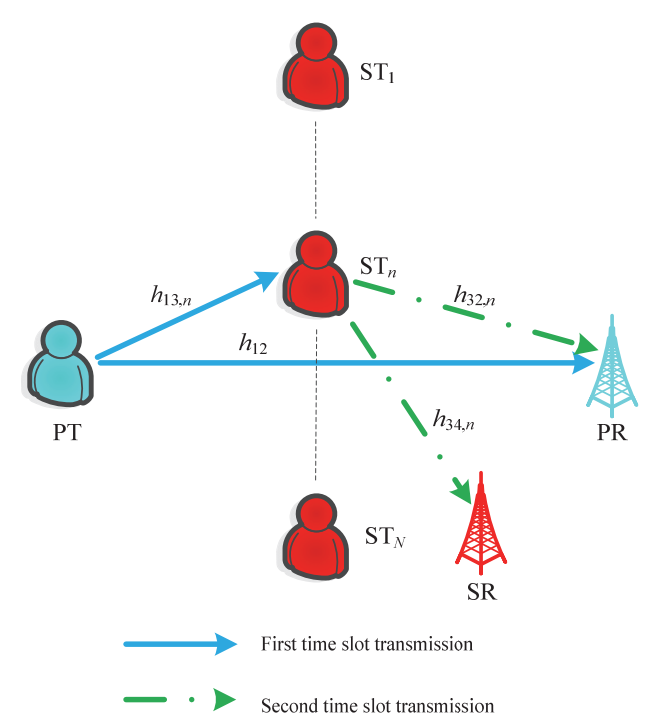

Fig. 10. System model with multiple secondary users

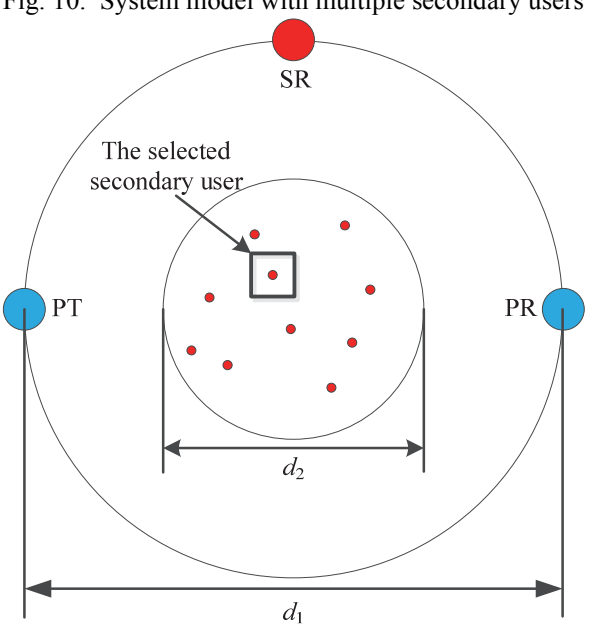

Fig. 11. Simulation scenario

terms of the outage probability of the secondary system with the optimal and sub-optimal solutions, which indicates the reasonability of aforementioned assumptions we made. Hence, we use the sub-optimal solution $\eta_{a}$ as the power allocation parameter to analyze the performance of our proposed spectrum sharing protocol in the following Section.

\section{Secondary User Selection Algorithm}

According to the analysis in the previous section, it is clear that the location of the secondary user participating in cooperation greatly influences the outage probability performance of both the primary and secondary systems. In order to further improve the performance of cooperative spectrum sharing; in this Section we analyze the secondary user selection algorithm of our proposed spectrum sharing protocol in the scenario with multiple secondary users as shown in Fig. 10.

From Fig. 7, it can be seen that when the distance of $\mathrm{PT} \rightarrow \mathrm{ST} d_{13}$ or $\mathrm{ST} \rightarrow \mathrm{PR} d_{32}$ is longer, i.e., the secondary user has bad channel conditions of PT $\rightarrow$ ST link or ST $\rightarrow$ PR link, the secondary user needs to allocate more power to assist the primary user so that the outage probability constraint of the
Table II Simulation Settings of Various Variables

\begin{tabular}{|c|c|c|}
\hline Variable & Definition & Simulation Settings \\
\hline$e$ & Signal separation error & $0.03,0.06,0.09$ \\
\hline$R_{p t}$ & Target rate of the primary user & $1 \mathrm{bit} / \mathrm{s} / \mathrm{Hz}$ \\
\hline$R_{s t}$ & Target rate of the secondary user & $1 \mathrm{bit} / \mathrm{s} / \mathrm{Hz}$ \\
\hline$v$ & Path loss exponent & 4 \\
\hline$\rho$ & $\begin{array}{l}\text { Guaranteed SNR threshold of the } \\
\text { primary user }\end{array}$ & $3 \mathrm{~dB}$ \\
\hline$N$ & $\begin{array}{c}\text { Number of available secondary } \\
\text { users }\end{array}$ & 1 to 17 , step is 4 \\
\hline$d_{1}$ & Radius of outer circle & 1 (normalized) \\
\hline$d_{2}$ & Radius of inner circle & 0.6 (normalized) \\
\hline$O_{\max }$ & $\begin{array}{l}\text { Maximum allowable outage } \\
\text { probability of the primary user }\end{array}$ & 0.02 \\
\hline$\sigma_{w}^{2}$ & $\begin{array}{c}\text { Power of additive white Gaussian } \\
\text { noise }\end{array}$ & $0.1 \mathrm{~W}$ \\
\hline$P_{p}$ & $\begin{array}{c}\text { Total transmit power of primary } \\
\text { user }\end{array}$ & $1 \mathrm{~W}$ \\
\hline$P_{s}$ & $\begin{array}{c}\text { Total transmit power of each } \\
\text { secondary user }\end{array}$ & $1 \mathrm{~W}$ \\
\hline$\xi$ & $\begin{array}{l}\text { Loss coefficient of the power } \\
\text { amplifier }\end{array}$ & 0.4 \\
\hline$P_{c s}$ & $\begin{array}{l}\text { Circuit power consumption of } \\
\text { secondary system }\end{array}$ & $0.05 \mathrm{~W}$ \\
\hline$P_{c p}$ & $\begin{array}{l}\text { Circuit power consumption of } \\
\text { primary system }\end{array}$ & $0.1 \mathrm{~W}$ \\
\hline
\end{tabular}

primary user can be satisfied. Moreover, the channel condition of the ST $\rightarrow$ SR link also has an important effect on the communication performance of the secondary system. Hence, in the proposed algorithm, each $\mathrm{ST}_{i}$ calculates a priority value that will be used to decide which secondary user to select for relaying; this priority value takes into consideration the distance of $\mathrm{PT} \rightarrow \mathrm{ST}_{i}$ links $d_{13, i}, \mathrm{ST}_{i} \rightarrow \mathrm{PR}$ links $d_{32, i}$ and $\mathrm{ST}_{i} \rightarrow \mathrm{SR}$ links $d_{34, i}$, respectively. The priority of each secondary user is thus computed by:

$$
M_{i}=\frac{1}{d_{13, i}} \frac{1}{d_{32, i}} \frac{1}{d_{34, i}}, i=1,2 \ldots N .
$$

where, $N$ is the number of secondary users in the cognitive radio network. As shown in the analysis of the system model above, the average channel gain can be reflected by the transmission distance.

From (43), it can be seen that the priority of secondary users decreases rapidly as the distance of ST $\rightarrow$ PR link increases, and hence, the secondary user with better channel condition of $\mathrm{ST} \rightarrow \mathrm{PR}$ link is more easily selected to assist the primary user. Moreover, the priority of secondary users also decreases with the distance of PT $\rightarrow$ ST link increasing. In this way, compared with other secondary users, the selected secondary user can use less power to assist the primary user to satisfy the outage probability requirement. Besides, the priority of secondary users also increases with the average channel gain of $\mathrm{ST} \rightarrow$ SR link and hence, a better communication performance of the secondary system can be obtained.

\section{Performance Analysis}

Before we analysis the performances of our proposed adaptive relaying mechanism, power allocation algorithm and relay selection algorithm, we list all the variables in simulation scenario as shown in Table II.

In this Section, we consider a round cell scenario as shown in Fig. 11. PT and PR are located at points $\left(-d_{1} / 2,0\right)$ and $\left(d_{1} / 2,0\right)$, 


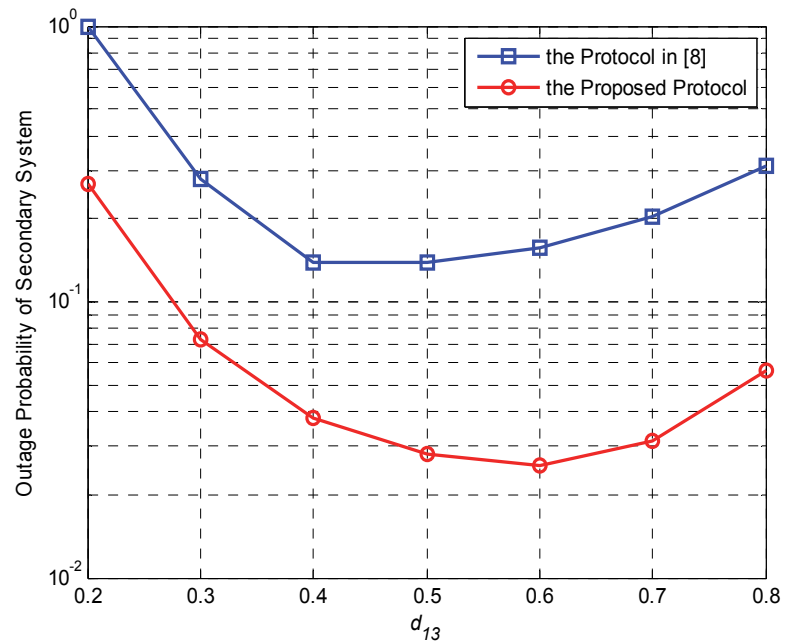

Fig. 12. Outage probability of the secondary system

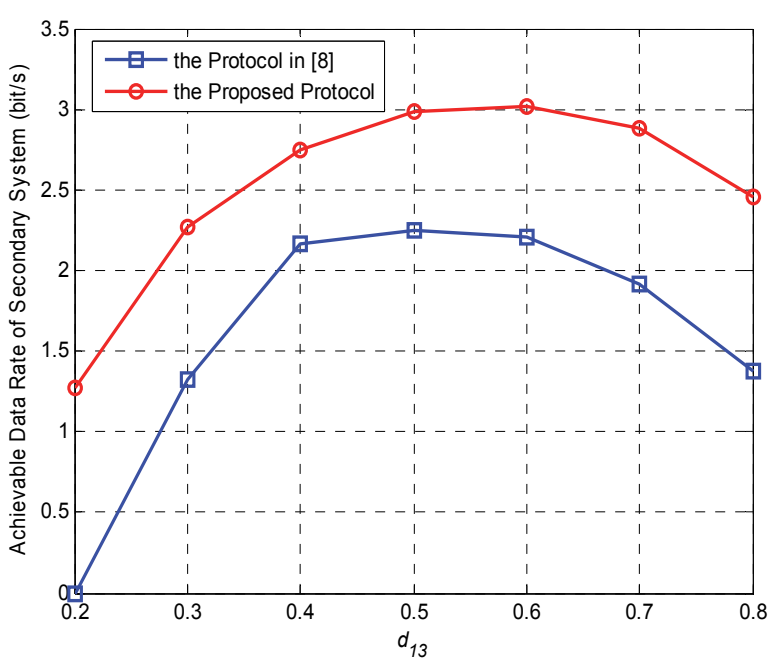

Fig. 13. Achievable data rate of the secondary system

respectively. SR is located at $\left(0, d_{1} / 2\right)$ and STs are randomly distributed in the central cell with the diameter of $d_{2}$.

When the distance of PT $\rightarrow$ ST link is either small or large enough, the primary user cannot satisfy its outage probability constraint even though ST uses the total power to forward the primary signal. In this way, any secondary users in the round cell with diameter $d_{2}$ can be selected to assist the primary user to achieve the outage probability constraint of the primary user. The performance of the proposed cooperative spectrum sharing protocol is compared with the spectrum sharing protocol in [8] and [10] as follows.

In [8], the secondary transmitter adopts the adaptive relaying mechanism and their goal is also to minimize the outage probability of secondary system. However, the locations of both primary and secondary users are fixed. In [10], they propose a relay selection strategy. Under the circumstance that plenty of ST are available to serve as the relay to help the transmission with primary user, the relay selection criteria is defined as $t_{1 i}=\Gamma_{1} / \gamma_{2 i}$, where $\Gamma_{1}=\sigma^{2} \rho_{p} t_{w} / P_{a}, \rho_{p}=2^{2 R_{p t}-1}$, and $\gamma_{2 i}$ is the estimated channel gains of $\mathrm{ST}_{i} \rightarrow \mathrm{PR}$ link, and $\sigma^{2}, t_{w}, P_{a}$ and $R_{p t}$ are all constant values. Almost the same way, when the timer reduced to zero first, the corresponding ST is

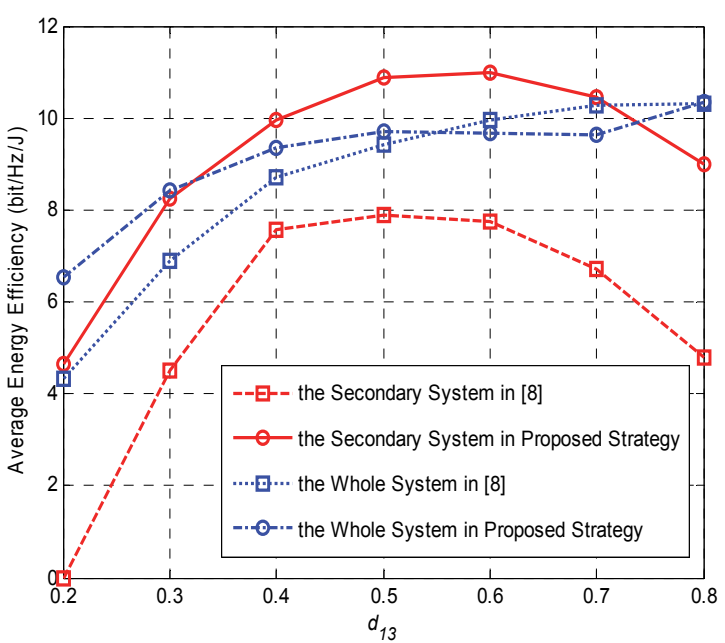

Fig. 14. Average Energy Efficiency against $d_{13}$

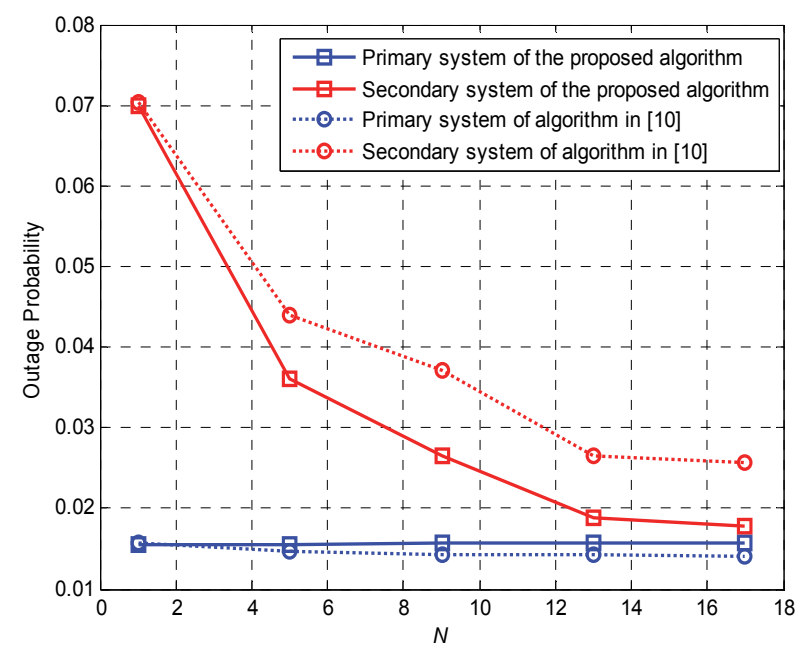

Fig. 15. The outage probability comparison

selected as the relay to assist primary users. In a word, they just select the relay according to the unique metric, the SNR of ST $\rightarrow$ PR link.

The performance of outage probability and achievable data rate for the secondary system with different locations of ST with the sub-optimal power allocation parameter $\eta_{a}$ in single secondary user environment is shown in Fig. 12 and Fig. 13, respectively.

We have considered the constraint that the maximum transmit power of each relay is restricted due to the reason that we consider the scenario that for each primary user, we can only select one relay to help the primary user transmitting its information.

Hence, with the number of relay increases, we have more opportunities to find a relay with both better ST $\rightarrow$ PR link and $\mathrm{ST} \rightarrow \mathrm{SR}$ link so that the achievable data rate of the above links can be improved, and that is the reason why the achievable data rate will always improve with the number of secondary user.

From Fig. 12 and Fig. 13, we can find that the outage probability of our proposed algorithm is much lower than that of algorithm in [8] for the secondary system. Moreover, approximately $33.3 \%$ achievable data rate gain can be obtained 


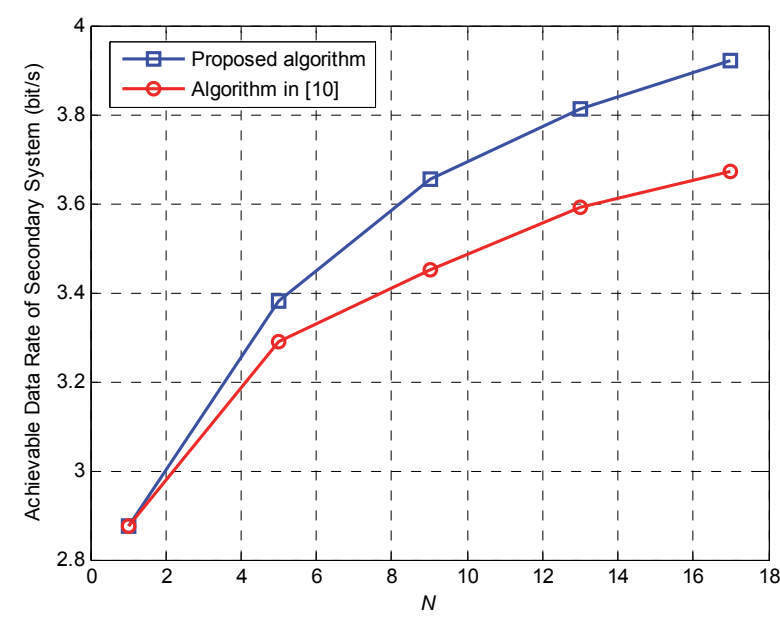

Fig. 16. The achievable data rate of secondary system comparison when compared with the algorithm in [8] when $O_{\max }$ is set to 0.02 . The great improvements of both outage probability and achievable data rate of the secondary system mainly attributes to two reasons. First of all, the secondary signal could be filtered from the primary signal through Fractional Fourier Transform domain filtering, which greatly reduces the interference from the primary system. Then, the secondary user could allocate less power compared with the algorithm in [8] to assist primary users by utilizing our proposed power allocation process while guaranteeing the outage probability of the primary users. In this way, when the transmit power of ST is fixed, the remaining power for secondary transmission goes up so that the promotion of achievable data rate and reduction of outage probability for secondary system could be achieved by utilizing our proposed power allocation process.

In this way, when the transmit power of ST is fixed, the remaining power for secondary transmission can be increased so that the outage probability of secondary system can be reduced and achievable data rate of secondary system can be increase. Moreover, due to the fact that ST can choose relay mechanism according to the decoding result of ST, ST still can assist the primary system with bad channel condition of PT $\rightarrow$ ST. Hence, secondary users can obtain more opportunities to access the spectrum, which is more flexible and suitable for different channel conditions.

From Fig. 14, we could find that the average energy efficiency of secondary system in our proposed strategy is better than that in [8]. The reason is that our strategy aims to minimize the outage probability of the secondary system. The power allocated to the primary system is just enough to guarantee the requirement of the primary user. Hence, more power is left for the secondary user so that an enhancement of average energy efficiency performance for the secondary system can be obtained. Besides, the average energy efficiency of the whole system is almost the same between our strategy and that in [8]. That is because the total transmit power in a complete transmission frame remains unchanged, and the power is used to transmit either the signal of secondary system or that of primary system in our proposed strategy and the strategy in [8].

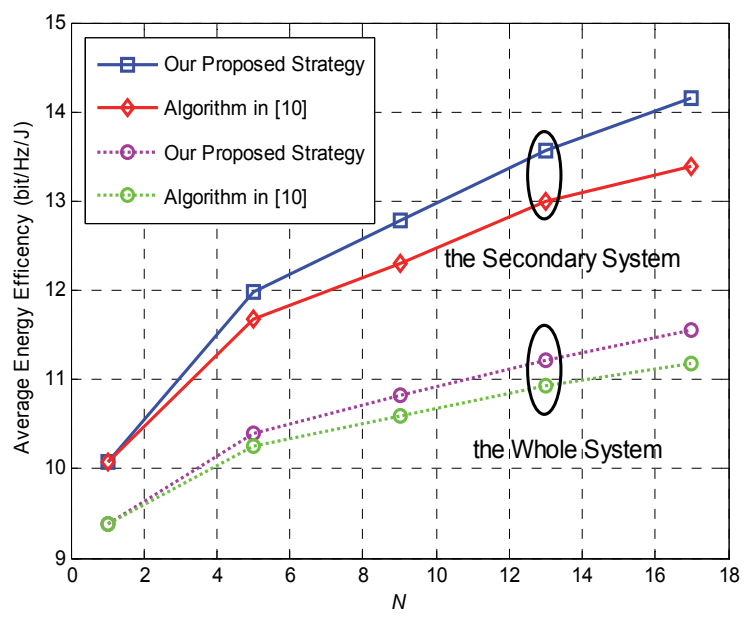

Fig. 17. Average energy efficiency versus the number of available secondary users $N$

In multiple alternative relays scenario, we compared three main metrics, i.e, outage probability, achievable data rate of secondary user, with that in ref. [10] when $O_{\max }$ is set to 0.02 . From Fig. 15, we could find that the outage probability of the secondary system is greatly reduced with higher number of secondary users. It is because the possibilities to find a better relay to increase the transmission data rate of the secondary user when more available relays exist. Moreover, the outage probability of the proposed protocol is lower than that in [10].

Although in [10], the authors use the instantaneous SNR of $\mathrm{ST} \rightarrow$ PR link as metric for relay selection, the channel status of $\mathrm{ST} \rightarrow \mathrm{SR}$ link is not considered which may result in lower transmission data rate of $\mathrm{ST} \rightarrow \mathrm{SR}$ link. In comparison, we combines the channel status of three links, i.e., PT $\rightarrow$ ST link, $\mathrm{ST} \rightarrow$ SR link and ST $\rightarrow$ PR link, to choose the best relay, and the comprehensive consideration of three links is helpful to reduce the outage probability of secondary system.

Although the outage probability of primary user in our protocol is a bit higher than that in [10], the value of outage probability for primary user is under the given threshold 0.02 , which satisfies the transmission requirement of primary user.

Fig. 16 illustrates the performance comparison of average achievable data rate of the secondary system. Since a better relay could be found with the increasing number of available relays, the achievable data rate is improved by $0.2 \mathrm{bit} / \mathrm{s}$ with normalized transmission bandwidth. Comparing with [10], since our relay selection strategy considers the channel status of three links, the most suitable relay could be selected to transmit its own signal as much as possible while guaranteeing the requirements of primary users. Therefore, a better performance of achievable data rate for the secondary system could be obtained.

According to the results shown in Fig. 17, when the number of available secondary users $N$ increases, the average energy efficiency for each system increases owing to the larger probability to select a better relay for both the primary system and the secondary system. Moreover, the average energy efficiency of the secondary user and the whole system in our proposed strategy are better compared with [10] due to the fact 
that our proposed relay selection strategy jointly considers three different links, i.e., PT-ST, ST-PR, and ST-SR.

\section{CONCLUSION}

In this paper, we propose a two-time slot transform domain processing based cooperative spectrum sharing protocol with adaptive relaying. In our proposed protocol, sinusoidal signals and chirp signals which can be easily distinguished through Fractional Fourier domain filtering are applied to primary and secondary users, respectively. Hence, the interference to both the primary and secondary systems can be greatly decreased. Moreover, secondary user acts as DF or AF relay adaptively according to channel conditions which makes the cooperation between the primary system and secondary system more flexible. Then in order to further improve the spectrum sharing performance, the optimization of power allocation scheme is analyzed to maximize the communication performance of the secondary system subject to the primary user's outage probability constraint being satisfied and a new secondary selection algorithm is proposed. Compared with the existing protocol, the proposed spectrum sharing protocol achieves a better performance in terms of outage probability, achievable data rate and average energy efficiency for the secondary system. Moreover, the performance of outage probability, achievable data rate and the average energy efficiency for the secondary system can be significantly improved by an increase in the number of secondary users.

Our current analysis focuses on single primary user scenario taking into account the optimization of power allocation and secondary user's selection algorithm. Our future work will be extended to the scenario with multiple primary users and multiple secondary users' selection. Besides, joint optimization of energy efficiency and spectrum efficiency is also a target we want to consider in the future.

\section{REFERENCES}

[1] R. C. Souza, J. R. A. Amazonas, and T. Abrao, "Power and Subcarrier Allocation Strategies for Energy-Efficient Uplink OFDMA Systems," in IEEE Journal on Selected Areas in Communications, vol. 34, no. 12, pp. 3142-3156, Dec. 2016.

[2] S. Bhattarai, J. M. Park. B. Gao, K. G. Bian, and W. Lehr, "An overview of dynamic spectrum sharing: ongoing initiatives, challenges, and a roadmap for future research," in IEEE Transactions on Cognitive Communications and Networking, vol. 2, no. 2, pp. 110-128, Jun. 2016.

[3] B. Wang and K. J. R. Liu, "Advances in cognitive radio networks: A survey," in IEEE Journal of Selected Topics in Signal Processing, vol. 5, no. 1, pp. 5-23, Feb. 2011.

[4] W. Jaafar, W. Ajib and D. Haccoun, "A new cooperative transmission scheme with relay selection for cognitive radio networks," in IEEE Global Communications Conference, Atlanta, GA, USA, 2013, pp. 949-954.

[5] L. Gao, L. J. Duan, and J. W. Huang, "Two-Sided Matching Based Cooperative Spectrum Sharing," in IEEE Transactions On Mobile Computing, vol. 16, no. 2, pp. 538-551, Feb. 2017.

[6] Y. Y. Pei, and Y. C. Liang, "Cooperative Spectrum Sharing with Bidirectional Secondary Transmissions," in IEEE Transactions on Vehicular Technology, vol. 64, no. 1, pp. 108-117, Jan. 2015.
[7] Y. Han, A. Pandharipande and S. H. Ting, "Cooperative decode-and-forward relaying for secondary spectrum access," in IEEE Transactions on Wireless Communications, vol. 8, no. 10, pp. 4945-4950, Oct. 2009

[8] A. Hyadi, E. M. Driouch, W. Ajib and M. S. Alouini, "Overlay cognitive radio systems with adaptive two-way relaying," in IEEE Global Communications Conference, Atlanta, GA, USA, 2013, pp. 937-942.

[9] R. Ou, W. J. Xu, S. Y. Li, and J. R. Lin, "Energy-Efficient Resource allocation for OFDM-based Cognitive Cooperation System Using Adaptive Relaying Strategy, " in IEEE Wireless Communications and Networking Conference (WCNC), Barcelona, Spain, 2016, pp. 984-989.

[10] Y. Han, S. H. Ting, and A. Pandharipande, "Cooperative Spectrum Sharing with Distributed Secondary User Selection," in IEEE International Conference on Communications, Cape Town, South Africa, 2010, pp. 1-5.

[11] Y. B. Li, Q. Y. Yin, W. Xu and H. M. Wang, "On the Design of Relay Selection Strategies in Regenerative Cooperative Networks with Outdated CSI," in IEEE Transactions on Wireless Communications, vol. 10, no. 9, pp. 3086-3097, 2011.

[12] T. Zhai, D. D. Liu, X. Y. Liu, et al, "Optimal Energy Efficient Strategy for Cooperative Spectrum Sharing Networks," in International Conference on Information and Communication Technologies, Kochi, India, 2014, pp. 1-5.

[13] R. F. Manna, F. S. Al-Qahtani, and S. A. Zummo, "A Full Diversity Cooperative Spectrum Sharing Scheme for Cognitive Radio Networks," in IEEE Access, vol. 5, pp. 17722-17732, 2017.

[14] M. Y. Wang, W. D. Lu, D. He, Z. J. Xu, and J. Y. Hua, "An Anti-Interference Spectrum Sharing Protocol with Secondary User Selection," in 10th International Conference on Communications and Networking in China (Chinacom), Shanghai, China, 2015, pp. 661-665.

[15] W. D. Lu, C. X. He, J. Y. Wu, H. Peng, and X. Liu, " Anti-Interference Cooperative Spectrum Sharing Based on Fairness Secondary User Selection," in International Wireless Communications and Mobile Computing Conference (IWCMC), Paphos, Cyprus, 2016, pp. 942-947.

[16] M. Babaei, E. Basar, and U. Aygolu, "A Cooperative Spectrum Sharing Protocol Using STBC-SM at Secondary User," in 24th Telecommunications Forum, Belgrade, Serbia, 2016, pp. 1-4.

[17] L. Lv, Q. Ni, Z. G. Ding, and J. Chen, "Application of Non-Orthogonal Multiple Access in Cooperative Spectrum Sharing Networks Over Nakagami-m Fading Channels," in IEEE Transactions on Vehicular Technology, vol. 66, no. 6, pp. 5506-5511, 2017.

[18] S. Jin, Y. Chen and S. Li, "Performance Analysis of a Dynamic Spreading Spectrum Strategy Based Spectrum Sharing for Cognitive Radio Networks," in Wireless Communications, Networking and Mobile Computing, Shanghai, China, 2012, pp. 1-5.

[19] X. Zhang and H. Su, "Opportunistic spectrum sharing schemes for CDMA-based uplink MAC in cognitive radio networks, " in IEEE Journal on Selected Areas Communications, vol. 29, no. 4, pp. 716-730, Apr. 2011.

[20] A. Serbes, and L. Durak, "A Centered DFT-Based Discrete Fractional Fourier Transform and Its Application to Chirp Signal Parameter Estimation," in 17th European Signal Processing Conference, Glasgow, Scotland, 2009, pp.1-5.

[21] Y. J. Zheng, C. Zhang, C. J. Li, Z. X. Zhu, and F. Wang, " Fractional Fourier Transform of Ultrasonic Chirp Signal for Range Measurement," in SICE Annual Conference 2015, Hangzhou, China, 2015, pp. 140-144. 
[22] J. Wu, Y. M. Jiang, and G. Y. Kuang, "Parameter Estimation for SAR Moving Target Detection Using Fractional Fourier Transform," in IEEE Geoscience and Remote Sensing Symposium, Quebec City, QC, Canada, 2014, pp. 596-599.

[23] X. L. Wu, N. N. Fu and F. Labeau, "Relay-Based Cooperative Spectrum Sensing Framework Under Imperfect Channel Estimation," in IEEE Communications Letters, vol. 19, no. 2, pp. 239-242, Feb. 2015.

[24] X. L. Wu, X. L. Han and F. Labeau, "Cooperative Spectrum Sharing Protocol Based on Transform Domain Processing with Joint Secondary Selection and Power Allocation," in International Wireless Communications and Mobile Computing Conference (IWCMC), , Paphos, Cyprus, 2016, pp. 475-480.

[25] E. Sejdic', I. Djurovic', L. Stankovic', "Fractional fourier transform as a signal processing tool: an overview of recent developments," in Signal Processing, vol. 91, pp. 1351-1369, 2011.

[26] R. Tao, Y. L. Li, and Y. Wang, "Short-Time Fractional Fourier Transform and Its Application," in IEEE Transactions on Signal Processing, vol. 58, no. 5, pp. 2568-2580, 2010.

[27] J. Shi, X. P. Liu, X. J. Sha, Q. Y. Zhang, and N. T. Zhang, "A Sampling Theorem for Fractional Wavelet Transform With Error Estimates," in IEEE Transactions on Signal Processing, vol. 65, no. 18, pp. 4797-4811, 2017.

[28] R. Tao, Y. L. Li and Y. Wang, "Short-Time Fractional Fourier Transform and Its Applications," in IEEE Transactions on Signal Processing, vol. 58, no. 5, pp. 2568-2580, May 2010.

[29] X. J. Sha, R. H. Wen and X. Qiu, "A new multiple-access method based on Fractional Fourier Transform," in Electrical and Computer Engineering CCECE '09, Canadian Conference on, St. John's, NL, Canada, 2009, pp. 856-859.

[30] V. Asghari and S. Aissa, "Performance of Cooperative Spectrum-Sharing Systems with Amplify-and-Forward Relaying," in IEEE Transactions on Wireless Communications, vol. 11, no. 4, pp. 1295-1300, Apr. 2012

[31] H. Peng, Y. Zhu, W. Lu, X. Liu and J. Hua, "Cooperative anti-interference spectrum sharing with secondary user selection," in China Communications, vol. 12, no. 9, pp. 45-52, 2015.

[32] J. Chen, L. Lv, Y. Y. Liu, Y. H. Kuo, and C. Ren, "Energy efficient relay selection and power allocation for cooperative cognitive radio networks," in IET Communications, vol. 9, no. 13, pp. 1661-1668, 2015.

[33] S. Shrestha and K. Chang, "Analysis of outage capacity performance for cooperative DF and AF relaying in dissimilar Rayleigh fading channels," in 2008 IEEE International Symposium on Information Theory, Toronto, ON, Canada, 2008, pp. 494-498.

[34] T. Sauer, "Numerical Analysis, " 2nd ed., the George Mason Univ. Press, 2012.

[35] J. N. Laneman, D. N. C. Tse, and G. W. Wornell, "Cooperative Diversity in Wireless Networks: Efficient Protocols and Outage Behavior," in IEEE Transactions on Information Theory, vol. 50, no. 12, pp. 3062-3080, 2004

[36] Y. Zhao, R. Adve, and T. J. Lim, "Improving Amplify-and-Forward Relay Networks: Optimal Power Allocation versus Selection," in IEEE Transactions on Wireless Communications, vol. 6, no. 8, pp. 3114-3123, 2007.

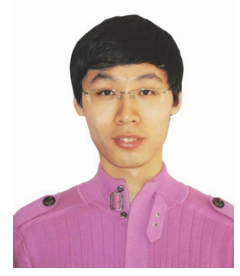

Xuanli Wu received his Ph.D. degree in communication and information system from Harbin Institute of Technology, Harbin, in 2008. He is currently an associate professor at the School of Electronic and Information Engineering, Harbin Institute of Technology, Harbin. He has served as the TPC member for many international conferences, including the IEEE ICC, GLOBECOM, VTC, INFOCOM, WCNC, PIMRC, WF-5G, WCSP, IWCMC. Also he is the Chair of IEEE Harbin VTS Chapter. He focuses on the fields of cooperative communications, ultra-dense networks, and non-orthogonal multiple access technology.

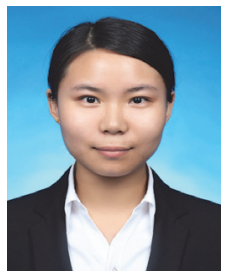

Xingling Han received her M.E. degree in information and communication engineering from Harbin Institute of Technology, Harbin, in 2016. She is currently an assistant engineer in the 30th Institute of China Electronics Technology Group Corporation. Her main research interests are cognitive radio networks.

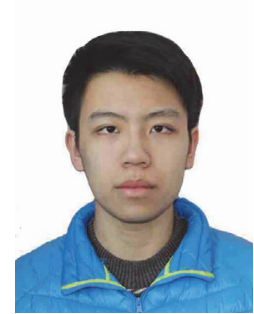

Xiao Deng received his B.E. degree in communication engineering from Harbin Institute of Technology, Harbin, in 2016 and he is currently a master candidate in Harbin Institute of Technology. He mainly focuses on cooperative spectrum sharing in $5 \mathrm{G}$ scenarios and MIMO technology.

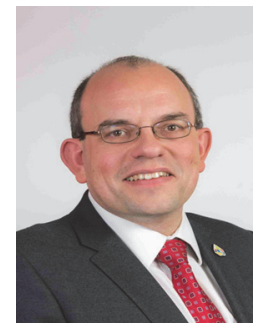

Fabrice Labeau is the Associate Dean (Faculty Affairs) in the Faculty of Engineering of McGill University, where he holds the NSERC/Hydro-Quebec Industrial Research Chair in Interactive Information Infrastructure for the Power Grid. His research interests are in applications of signal processing to healthcare, power grids, communications and signal compression. He has authored more than 175 refereed papers in refereed journals and conference proceedings in these areas.

$\mathrm{He}$ is the associate director for operations of STARaCom, an interuniversity research center grouping 50 professors and 500 researchers from 10 universities in the province of Quebec, Canada. He is junior past president of the Institute of Electrical and Electronics Engineers (IEEE) Vehicular Technology Society, President-Elect of the IEEE Sensors Council, and the past chair of the Montreal IEEE Section. He was a recipient in 2015 and 2017 of the McGill University Equity and Community Building Award (team category), of the 2008 and 
2016 Outstanding Service Award from the IEEE Vehicular Technology Society and of the 2017 W.S. Read Outstanding Service Award form IEEE Canada.

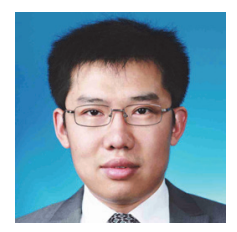

Shuai Han received his ME and PH.D degree in Information and Communication Engineering from Harbin Institute of Technology in 2007 and 2011, respectively. And he completed his post-doctoral work in 2012 in Electrical and Computer Engineering from Memorial University of Newfoundland in Canada. He is currently an associate professor at Department of Electronics and Communication Engineering, Harbin Institute of Technology. His research interests include wireless sensor networks, wireless communications, the global navigation satellite system and indoor location. He is an associate editor of IEEE Access and Journal of Communications and Information Networks (JCIN). He has served as a co-chair for technical symposia of international conference ICC 2018, VTC FALL 2016, IWCMC 2017 IWCMC 2016, IWCMC 2015, WiCON 2017. He has also served as the TPC member for many international conferences, including the IEEE ICC, IEEE GLOBECOM, VTC, IEEE COMNETSAT, APCC. Also he is senior member of IEEE Communication Society, Vice Chair of IEEE Harbin ComSoc Chapter and Vice Chair of IEEE Harbin VTS Chapter. 